\title{
The functional organization of area V2, I: Specialization across stripes and layers
}

\author{
STEWART SHIPP AND SEMIR ZEKI \\ Wellcome Department of Cognitive Neurology, University College, Gower Street, London WC1E 6BT, UK
}

(Received December 28, 2000; AcCEPTEd February 15, 2002)

\begin{abstract}
We used qualitative tests to assess the sensitivity of 1043 V2 neurons (predominantly multiunits) in anesthetised macaque monkeys to direction, length, orientation, and color of moving bar stimuli. Spectral sensitivity was additionally tested by noting ON or OFF responses to flashed stimuli of varied size and color. The location of 649 units was identified with respect to cycles of cytochrome oxidase stripes (thick-inter-thin-inter) and cortical layer. We used an initial 8-way stripe classification (4 stripes, and 4 "marginal" zones at interstripes boundaries), and a 9-way layer classification (5 standard layers (2-6), and 4 "marginal" strata at layer boundaries). These classes were collapsed differently for particular analyses of functional distribution; the main stripe-by-layer analysis was performed on 18 compartments ( 3 stripes $\times 6$ layers). We found direction sensitivity only within thick stripes, orientation sensitivity mainly in thick stripes and interstripes, and spectral sensitivity mainly in thin stripes. Positive length summation was relatively more frequent in thick stripes and interstripes, and negative length/size summation in thin stripes. All these "majority" characteristics of stripes were most prominent in layers 3A and 3B. By contrast, "minority" characteristics (e.g. spectral sensitivity in thick stripes; positive size summation in thin stripes) tended to be most frequent in the outer layers, that is, layers 2 and 6 . In consequence, going by the four functions tested, the distinctions between stripes were maximal in layer 3, moderate in layer 2, and minimal in layer 6. Pooling all layers, there was some indication of asymmetry in the stripe cycle, in that thin stripe characteristics (spectral sensitivity, orientation insensitivity, and negative size summation) were also evident in the marginal zone and interstripe immediately lateral to a thin stripe, but less so medially. Within thin stripes, spectral and orientation selectivities were negatively correlated; this was still more accentuated amongst the minority spectrally tuned cells of thick stripes, but absent from interstripes, where these two properties were randomly assorted. Directional and spectral sensitivities were each coupled to negative size summation, but not to each other. We conclude that these functional characteristics of stripes are consistent with segregated, specialized pathways ascending through their middle layers, whilst the outer layers, 1, 2, and 6, utilize feedback from higher areas to adopt a more integrative role.
\end{abstract}

Keywords: Cytochrome oxidase stripes, Cortical lamination, Ascending pathways, Feedback, Functional specialization, Functional integration

\section{Introduction}

The modular construction of area V2 is one of the most arresting features of cortical organization that has yet been discovered (Livingstone \& Hubel, 1982; Tootell et al., 1983). These modules (stripes) are vehicles for functional specialization (Roe \& Ts'o, 1997), yet V2 is also figured to play a role in integrating separate visual functions, and the evidence supporting segregation of function on one hand, and integration on the other, has been ambivalent (Levitt et al., 1994a; Gegenfurtner et al., 1996; Tamura et al., 1996). In this paper, we propose a resolution to this impasse, that arises from a thorough study of the distribution of functions across

Address correspondence and reprint requests to: Stewart Shipp, Department of Cognitive Neurology, Darwin Building, University College, Gower Street, London WC1E 6BT, UK. E-mail: s.shipp@ucl.ac.uk cortical layers as well as stripes. The functional characteristics of layers are best known by their status with regard to ascending and descending patterns of cortical connectivity (Felleman \& Van Essen, 1991). The present findings suggest a physiological analogue of this anatomical pattern, that stripes are clearly distinct from each other in their middle-upper layers (layers 4 \& 3) carrying the ascending pathway, and less so in the outer layers $(1 / 2$ \& 5/6) which receive feedback: in other words, that segregation and integration are separated across layers in V2.

The first hint of functional periodicity in V2 was provided by fluctuations in the density of pulvinar afferents (Benevento \& Rezak, 1976; Ogren \& Hendrickson, 1977; Curcio \& Harting, 1978; Lund et al., 1981), but it was only with the advent of cytochrome oxidase staining that the regular cyclic, striped organization became apparent (Livingstone \& Hubel, 1982). This led to a rapid exposition of the specific connectivity and physiology of 
the stripes (Livingstone \& Hubel, 1983; DeYoe \& Van Essen, 1985; Hubel \& Livingstone, 1985, 1987; Shipp \& Zeki, 1985), giving rise to the general interpretation that the V2 stripes are a key component of the segregated, specialized pathways that originate in V1 and distribute differentially across prestriate cortex (DeYoe \& Van Essen, 1988; Livingstone \& Hubel, 1988; Zeki \& Shipp, 1988). Ironically, it is likely to be the pulvinar inputs, more than anything else, that are directly responsible for the cycles of cytochrome oxidase density, but their specific function is still unknown (Livingstone \& Hubel, 1982; Wong-Riley \& Carroll, 1984; Levitt et al., 1995).

In many respects, the physiological properties of thin stripes, interstripes, and thick stripes follow the specializations of their sources in V1-respectively blobs and interblobs in layer 2/3 and layer 4B. Blobs and thin stripes contain unoriented, wavelengthselective cells preferring low spatial frequencies, whilst interblobs and interstripes demonstrate the opposite features (Livingstone \& Hubel, 1984a; DeYoe \& Van Essen, 1985; Hubel \& Livingstone, 1987; Tootell et al., 1988b, 1998c; Ts'o \& Gilbert, 1988; Tootell \& Hamilton, 1989; Zeki \& Shipp, 1989a; Ts'o et al., 1990; Levitt et al., 1994a; Malach et al., 1994; Edwards et al., 1995; Roe \& Ts'o, 1995). Layer 4B of V1 and V2 thick stripes share selectivities for orientation, disparity, and direction (Dow, 1974; DeYoe \& Van Essen, 1985; Zeki \& Shipp, 1989a; Hubel \& Livingstone, 1990; Levitt et al., 1994a; Munk et al., 1995; Roe \& Ts'o, 1995). For shorthand purposes, this means that thin stripes, interstripes, and thick stripes, respectively, contribute to perceptual attributes of color, static form, and motion/depth/dynamic form. There are also many parallels that can be drawn with the physiology of their immediate targets in prestriate cortex, V4 receiving output from thin stripes and interstripes, V3 and V5 receiving output from thick stripes (DeYoe \& Van Essen, 1985; Shipp \& Zeki, 1985; Shipp \& Zeki, 1989b; Zeki \& Shipp, 1989b; Nakamura et al., 1993; DeYoe et al., 1994; Munk et al., 1995; Felleman et al., 1997). All of this evidence supports the notion of segregated, functionally specialized pathways coursing through V2-but yet does not provide a complete picture.

The counterpoint to this picture of functional specialization is that area V2 also plays an important role in allowing its constituent pathways to communicate. The reasoning at first was circumstantial: what could be the utility of a second, multipurpose area, interposed between V1 and more narrowly specialized areas of prestriate cortex, composed of an anatomically rearranged but physiologically similar trio of compartments-unless it is to facilitate interactions between them? (Shipp \& Zeki, 1989b; Roe \& Ts'o, 1995). More recently, evidence consistent with information exchange between the pathways has emerged. The intrinsic circuitry in V2 traverses all three stripe systems (Levitt et al., 1994b), selective responses to the same attribute may be found in all compartments (Peterhans \& von der Heydt, 1993; Levitt et al., 1994a; Gegenfurtner et al., 1996), and independent of stripe identity, joint selectivities for pairs of attributes might be randomly assorted (Burkhalter \& Van Essen, 1986; Gegenfurtner et al., 1996; Tamura et al., 1996). Previous discussion of these findings has cast them in terms of "integration", in the sense that the stripes' segregation of properties might be less complete than previously thought, and their relative specialization thus less prominent (Levitt et al., 1994a; Gegenfurtner et al., 1996; Tamura et al., 1996). Integration is a broad term, applicable to any form of information exchange between functionally distinct layers, modules, areas, etc. Here, the integration may serve "cue-invariance," that is, the use of multiple sensory cues to establish a single particular perceptual attribute (DeYoe \& Van Essen, 1988; Albright, 1992; Sary et al., 1993). In pursuing the notion that stripes desegregate cues, Gegenfurtner et al. (1996) conclude that V2 "achieves a cueinvariant representation of the visual world," without allowing that the object of the representation (i.e. the perceptual attribute that is being cued) might vary across stripes. If so, this is a form of integration in V2 that may actually facilitate specialization of function. Other forms may, potentially, subserve "binding" of specialized elements. Although these are issues that we cannot expect to resolve fully with the present (mainly multiunit) data, they are the natural avenues by which to approach its interpretation.

In summary, the aim of the present paper is two-fold: firstly, to present a large body of qualitative data on specialized response selectivities across V2 stripes, that was incomplete in previous reports (Shipp \& Zeki, 1985; Zeki \& Shipp, 1989a); secondly, to augment the stripe classification with an analysis of cortical lamination, to provide a functional breakdown within a fully threedimensional (3D) anatomical compartmentalisation of V2. This has been attempted before, but with an insufficient sample size of units resulting, even at low resolution, in fragmentary and inconsistent conclusions (Levitt et al., 1994a; Gegenfurtner et al., 1996); (e.g. in thick stripes, these studies respectively nominate the middle, or deep layers as the most prominent source of directionselective units). In practical terms, we found that the identification of stripes and layers was usefully facilitated by digital image processing, helping to amplify the previous hand-drawn sketches. We undertook the laminar analysis in view of the fact that different forms of integration may be achieved over forward, intrinsic and feedback pathways that observe different laminar patterns of connectivity (Zeki \& Shipp, 1988; Felleman \& Van Essen, 1991). We found a laminar physiological organization to reflect the anatomical one, and this provided the key for reconciling our stripe data and several earlier, mutually inconsistent reports, within a picture of complementary segregative and integrative processes at work in $\mathrm{V} 2$.

\section{Methods}

\section{Experimental protocols}

We report data obtained from a total of 14 juvenile $M$. fascicularis. Standard means were used to prepare animals for terminal sessions of electrophysiological recording, 1-3 days duration (Zeki, 1974). Anesthesia was induced with ketamine (Vetalar, $10 \mathrm{mg} / \mathrm{kg}$, i.m.) for insertion of cannulae, and maintained with pentobarbitone (Sagatal, 2-3 mg/kg/h, i.v.) for cranial surgery and recording. Ocular movements were minimized with pancuronium bromide (Pavulon, $0.5 \mathrm{mg} / \mathrm{kg} / \mathrm{h}$, i.v.). The animals were artificially ventilated through a tracheal cannula using room air, the rate and volume being regulated to maintain expired $\mathrm{CO}_{2}$ at around $4-4.5 \%$. Rectal temperature was maintained at $37^{\circ} \mathrm{C}$ using a thermostatic blanket. Pupils were dilated with topical application of atropine, and protected with neutral contact lenses. Auxiliary lenses were used to refract the eyes on to a tangent screen at a distance of $114 \mathrm{~cm}$. Foveal locations on the screen were determined by a reversible ophthalmoscope, and replotted after periodic removal of contact lenses for cleaning and irrigating the eyes with saline.

The skull was opened over the midline, and a small $(5 \mathrm{~mm})$ window made in the dura over the lunate sulcus near the hemispheric midline. The electrode was positioned just over the cortex posterior to the sulcus and angled parallel to it, pointing laterally and as acute as possible to the cortical surface. The defect was then 
sealed with agar; we did not use a chamber, to avoid restriction of access. Due to the angle of entry, the majority of recordings were made beneath intact dura, with generally good stability. The electrode was advanced until the first neural activity was encountered, and then at steps of 50-200 $\mu \mathrm{m}$ between cells (most commonly in regular steps of $100 \mu \mathrm{m}$ in order to cover as much territory as possible while still permitting a local analysis of topography). Occasional minor adjustments were made to isolate a single spike, but the great majority of recording sites were multiunits. Neural activity was monitored over a loudspeaker. The animal faced a translucent tangent screen, covered with white paper at the current site of plotting. Background illumination, to maintain mesopic light adaptation, was provided by a $60-\mathrm{W}$ room lamp. This was routinely extinguished during receptive-field plotting and for some stimulus testing. Rectangular receptive fields were plotted with a tungsten filament, 150-W handheld projector, using bars or spots of light, and with resort to color filters or manual dark bars if necessary.

Experiments were terminated with a lethal dose of anesthetic and the brains processed by standard methods, described previously (Shipp \& Zeki, 1989a,b). Briefly, the occipital operculum was removed, flattened, and cut tangentially at $50 \mu \mathrm{m}$, to generate sections passing roughly parallel to the layering of V2 in the posterior banks of the inferior occipital and lunate sulci. All sections were stained for cytochrome oxidase (Wong-Riley, 1979). The stain was found to provide a sensitive background for the detection of electrode tracks and lesions (Hubel \& Livingstone, 1987).

\section{Classification of response selectivities}

Once the field was plotted, one eye was used for further testing. We assessed response selectivity to the orientation, direction, length, and wavelength composition of a bar stimulus, moving perpendicular to its long axis. The tests were invariably qualitative, and governed by the auditory monitor of spike activity. Reference to recorded output from the spike discriminator and chart recorder was available if required. For each variable, cells were placed in the following categories.

- Orientation : selective-one orientation (i.e. axis of motion) is maximal and there is no reliable response to the orthogonal orientation; bias - a reliable difference in response to the best and orthogonal orientations; unselective-otherwise.

- Direction : selective-one direction is maximal and there is no response to the opposite direction; bias - a reliable difference in response to the best and opposite directions; unselectiveotherwise.

- Length (area) summation : facilitated - a bar extending beyond the field dimensions gives an enhanced response, or a bar of field size is a better stimulus than a spot; antagonistic - a bar extending beyond the field dimensions gives a reduced response, or a bar of field size is a poorer stimulus than a spot; tuned-if a bar of approximately field size gives greater activity than either a longer or shorter bar (or spot); unselective-otherwise. For units lacking orientation selectivity, the bars were often broadened in width, so yielding equivalent categories of area summation.

- Wavelength sensitivity: the tests utilized gelatin filters with peak transmittances at 660, 570, or $445 \mathrm{~nm}$ (LW, MW, \& SW, respectively). Units were screened with moving colored bars, and then scrutinized with static flashed stimuli to assess differ- ential ON or OFF responsivity. Cells were classed as narrowband selective - an ON response to one filter, an OFF response to at least one other, and a reliably weaker, or absent response to white; narrowband bias - an ON (or OFF) response to one filter only and an equal response to white; broadband bias-selective (ON) responses to two filters, with an equal response to white light, and absent (or weak OFF) responses to the third. A final category was dark-selectivity, responding only to dark stimuli on a light ground, and/or producing only OFF responses to light of any wavelength. Most commonly, broadband bias units were those which lacked a response to the SW filter. Before reaching this conclusion, the room lamp was extinguished to boost the contrast of the SW stimulus.

It may be noted that the criteria for orientation and direction criteria, hinging on orthogonal or opposite directions of bar motion, are formally independent: thus, a directional-selective unit might, in principle, be classified as nonoriented if it displayed equal sensitivity to a 180-deg range of directions and a null over the opposite 180-deg. In practice, no units with such a "half-moon" tuning curve were found. The color filters were those used for previous studies of the responses of V4 cells to multicolored "Mondrian" displays (Zeki, 1983). They were initially selected such that the Mondrian display adopted a monochromatic appearance under illumination through each filter (reddish, yellowishgreen, or bluish). They are also appropriate for chromatic screening tests because, physiologically, this criterion depends on the transmitted waveband of each filter avoiding the spectral loci of unique hues - the crossover points for opponent-color mechanisms. Thus, each filter activates just one chromatic class of cell at retinal or geniculate levels - that is, R-G, G-R, and B-(R\&G) (Derrington et al., 1984), respectively, for the LW, MW, and SW filters. At a cortical level, the MW filter will selectively activate "yellowblue," as well as "green-red" opponent cells (Livingstone \& Hubel, 1984a; Ts'o \& Gilbert, 1988); however, it was not our goal to study color mechanisms per se, or seek to individuate specific color pathways whose separate existence at cortical levels is more contentious (Lennie et al., 1990; Kiper et al., 1997; De Valois et al., 2000).

\section{The identification of cell position in relation to metabolic architecture and layer}

Thick and thin stripes were identified by their relative width and the principle of alternation (Livingstone \& Hubel 1982); although this initial description, pertaining to squirrel monkeys, was subsequently questioned in macaques (Hubel \& Livingstone 1987), other experience has been that the majority of stripes in the majority of brains are readily classifiable (Shipp \& Zeki, 1989b; Tootell \& Hamilton, 1989; Zeki \& Shipp, 1989b; Olavarria \& Van Essen, 1997). The absolute width of a single stripe may be a poor indicator (since the widest thin stripe may exceed the narrowest thick stripe), but when a series of stripes is visualized in the flattened occipital operculum, regular alternation in relative width is normally apparent. Density of staining is not always a reliable means of distinction, although some thick stripes may be lighter than average, and some thin stripes darker-often these have a narrow central spine with prominent cross striations. As we noted previously (Shipp \& Zeki, 1989b), there are occasional local irregularities where dark stripes branch, or merge. In these instances provisional "thick" or "thin" character may be assigned by extrapolating from the alternation visible in neighboring regions. 
Electrode tracks through the stripes were reconstructed by reference to lesions, and points of transition between cortex and white matter. Even in the most accurate cases (e.g. Fig. 1, where all four lesions occur in a single section), it is impossible to locate individual recording sites to a precision better than $\pm 50 \mu \mathrm{m}$ (with lesions of width up to $400 \mu \mathrm{m}$ ). This is also about the maximal resolution that can be achieved in plotting the borders of the most sharply demarcated cytochrome stripes. In less optimal cases the

(a)

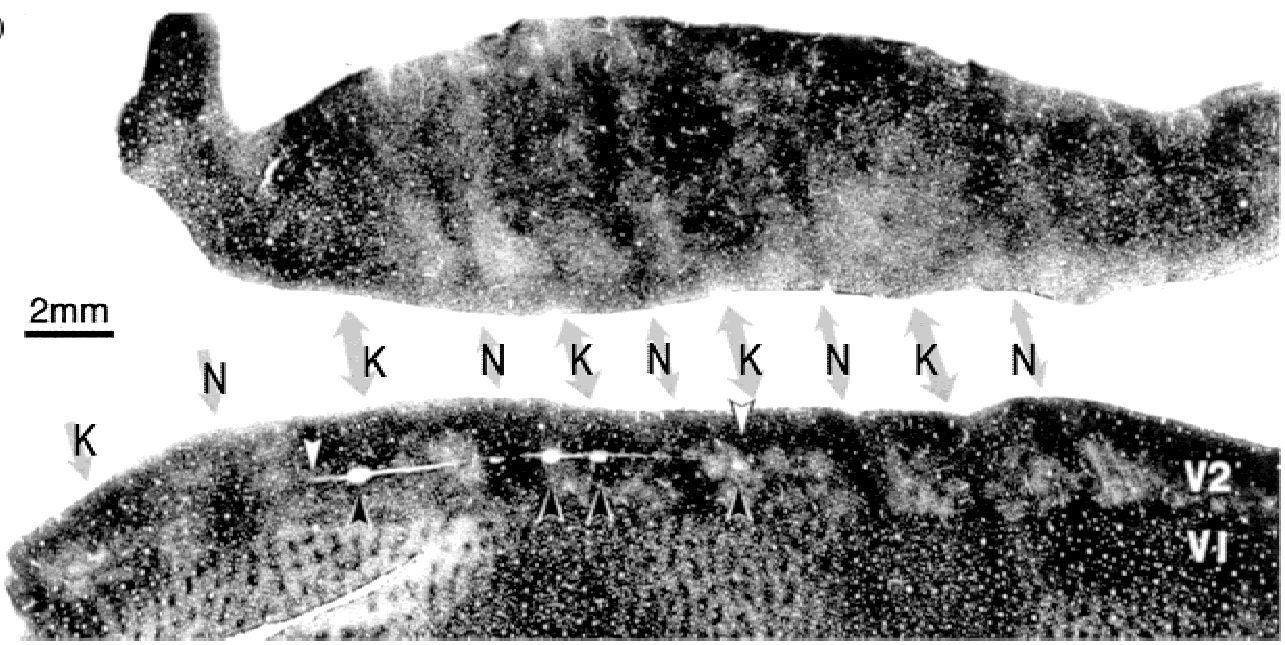

(b)
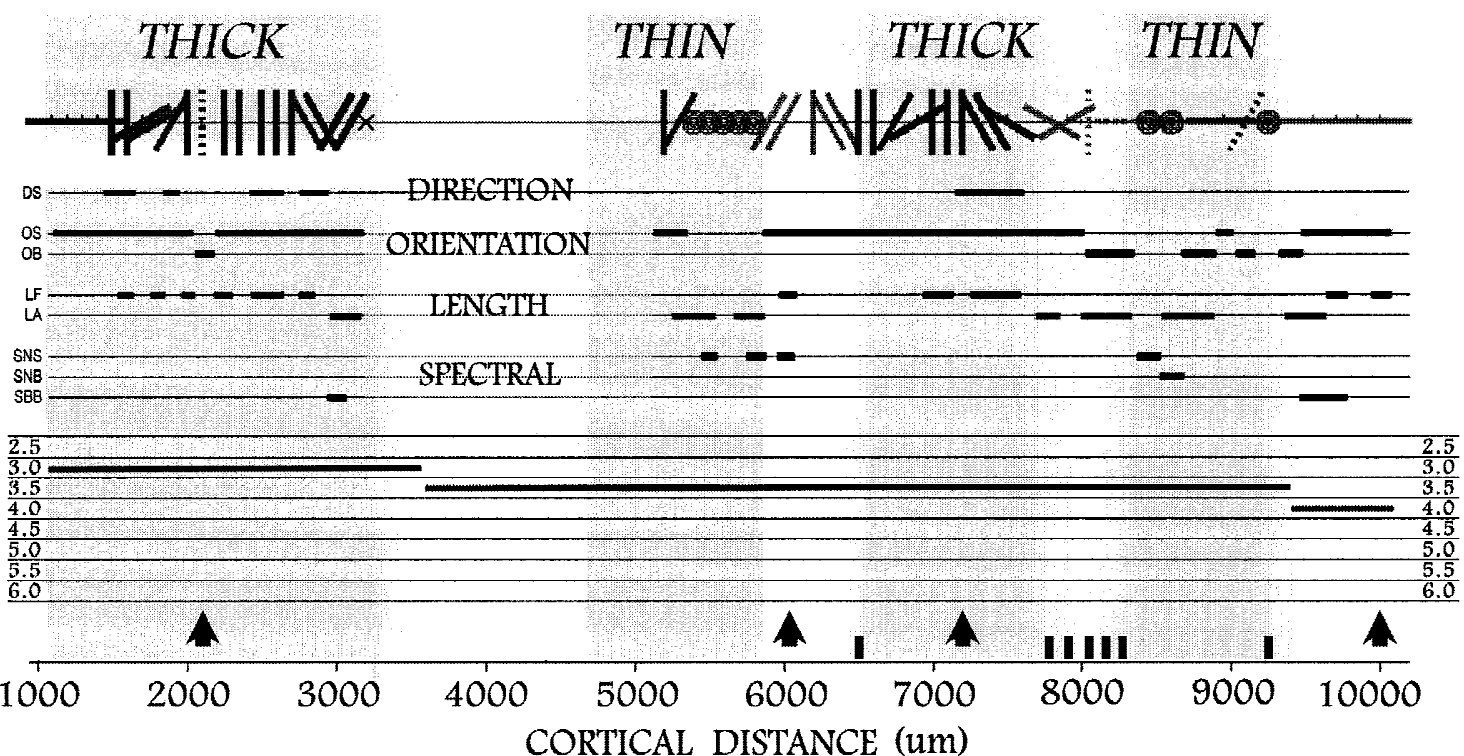

Fig. 1. (a) Electrode track with 60 units recorded across $9 \mathrm{~mm}$ and two complete stripe cycles of V2 (case SP07). The direction of travel was from medial-to-lateral (rightward). The first and last recorded units are marked by white arrowheads; dark arrowheads indicate track-marking lesions. This track was made in the external margin of V2, bordering V1; to facilitate stripe identification, a section through the posterior bank of the lunate sulcus is shown above, inverted, so that individual stripes can be traced between the two sections; K: thick, N: thin. From the cytochrome pattern in V2 (and also in neighboring V1), the first lesion can be judged to fall within layer 3, and the fourth in layer 4. The second and third lesions are classed as " 3.5 ," indeterminate between layers 3 and 4. (b) Summary of the categorization of physiological response properties encountered along the electrode track. The horizontal axis is recording depth $(0=$ cortical surface), with the location of lesions marked by arrows, and histological marginal units by small vertical ticks on the baseline. The locations of dark stripes are identified by the fuzzy grey bands labeled thick and thin, whose diffuse borders correspond to the local dispersion of marginal units. Cortical layer is shown above, followed by physiological properties. Spectral sensitivity: SBB — broadband biased; SNB — narrowband biased; SNS—narrowband, or dark, selective. Length sensitivity: LA—antagonized or tuned; LF—facilitated. Orientation sensitivity: OB-biased; OS—selective. Direction: DS—direction sensitive (i.e. selective or biased). The top line shows each unit's preferred orientation; selective and biased units are coded by solid and dashed bars, respectively, unselective cells by circles. There is a gap of $2000 \mu \mathrm{m}$ from cell 21 at $3200 \mu \mathrm{m}$ to cell 22 at 5200 (rapid advance of the electrode was occasioned by the onset of small, seemingly monocular RF's at $3300 \mu \mathrm{m}$, mistaken to indicate that the electrode had passed from V2 into V1). Thus, no units were recorded in the margins of the first I stripe; the N/I border just before 6000 is comparatively sharp so the first unit classed as a histological marginal is $\mathrm{I} / \mathrm{K}$ at a depth of 6500 . The subsequent I stripe is locally obscured by the neighboring $\mathrm{K}$ and $\mathrm{N}$ stripes, resulting in a string of $\mathrm{K} / \mathrm{I}$ and $\mathrm{I} / \mathrm{N}$ marginals (the $\mathrm{K}$ and $\mathrm{N}$ stripes can be seen to retain separate identities in V2 more anteriorly, so this is a local conjugation, rather than a merger, of the two stripes). The final stripe border has one N/I unit. 
electrode track may traverse many sections, lesions may be large or unidentifiable, or the stripe borders more blurred. Due to these factors, we were unable to classify all recording sites unambiguously, resorting instead to the following categories: $\mathbf{K}, \mathbf{K} / \mathbf{I}, \mathbf{I}^{\mathbf{m}}$, $\mathbf{I} / \mathbf{N}, \mathbf{N}, \mathbf{N} / \mathbf{I}, \mathbf{I}^{\mathbf{l}}$, and $\mathbf{I} / \mathbf{K}$. These classes are ordered as a medialto-lateral sequence through one stripe cycle; thus, for example, $\mathrm{K} / \mathrm{I}$ refers to the marginal zone between a thick stripe and the interstripe which is lateral to it, and $\mathrm{I}^{\mathrm{m}}$ refers to an interstripe bordered by a thick stripe medially and a thin stripe laterally. Strictly, the marginal zones should not be taken as anatomically defined territories: they are just a residue of recording sites with indeterminate stripe affiliation.

We followed a similar strategy in respect of the cortical layering, assigning each recording site to one of the following categories:$2,2.5,3,3.5,4,4.5,5,5.5$, and 6 . Again 2.5 implies that a recording site was near to the border between layers 2 and 3 , and 3.5 between 3 and 4, etc. Layers were identified from the cytochrome stain in the majority of cases: the superficial layers stain more heavily, and the dark/light junction running about midway through the cortical thickness corresponds to the layer 3B/4 border; within the dark stripes this junction is slightly more diffuse because upper layer 4 may also stain heavily. Layer 5 is the palest layer, and layer 6 is sufficiently darker to recognize the $5 / 6$ border in average quality stains. Cortical entry and exit points (i.e. pial surface and white matter) were also noted. As electrodes were angled obliquely to the cortex the passage through all six layers was generally several $\mathrm{mm}$, enabling laminar estimates for almost all the electrode tracks that could be recovered.

\section{Statistical procedures}

A total of 649 recording sites were classified in both anatomical dimensions (i.e. stripe and layer), and the great majority of these were also classified in each of the four physiological dimensions. We examined the data for certain binary and ternary interactions between these factors, using the $G$-statistic to fit a log-linear model (Sokal \& Rohlf, 1995). The procedure tests first for the presence of 3-way interaction: if positive, it uses separate tests to examine how the 2-way interaction between a particular pair of factors may vary at each level of the third factor; if 3-way interaction is rejected, it tests for a particular 2-way interaction pooled across all levels of the third factor. The latter test reveals the real, underlying contingency between the first two factors, irrespective of any relationship each might have with the third factor.

\section{Results}

\section{Discrimination of stripes and layers}

Figs. 1-4 illustrate six penetrations from four separate cases. Ideally, stripes can be identified in sections that contain the electrode track and that cut tangentially across V2 (parallel to the layers), either in the external margin that abuts V1 or, more rarely, in the larger portion buried inside the lunate sulcus. The external margin of V2 may be only 1-2 mm wide, but this can be sufficient to see stripes. The pattern is generally clearer inside the sulcus, and any ambiguity can be resolved by aligning the two samples, since the stripes run continuously (e.g. Fig. 1); if the external rim of V2 is abnormally wide, this may not be necessary (e.g. Fig. 2). Less helpfully, the electrode track may be recovered from sections that pass radially (i.e. perpendicular to the layers) through V2 on the tip of the gyrus, and then into the white matter underlying V1. Stripes cannot be identified with any great reliability from radial transects, and it is necessary to superimpose the pattern with the nearest tangential section from the lunate sulcus (e.g. Fig. 3). The replacement of hand-drawn sketches by digital image processing has made this procedure more efficient.

Stripes are irregular, diffuse creatures with a variable blob-like or reticular substructure (Wong-Riley \& Carroll, 1984). Thus, even with perfect histology, the exact placement of a border has an arbitrary element. Take for example Fig. 4, showing a track through V2 in the lunate sulcus with two lesions less than $2 \mathrm{~mm}$ apart. The first lesion is on the edge of a thin stripe with prominent cross striations (not an unusual feature-see Fig. 3 for another example). The boundary of this stripe might be taken as straight or sinuous. The track crosses the interstripe at a point where it seems relatively narrow, proceeding into a protuberance on the adjacent thick stripe. Because of such features, it is possible that 200$300 \mu \mathrm{m}$ stretches of track belong to ambiguous zones; to minimize arbitrary choices, we have classified units recorded in these zones as marginal (e.g. $\mathrm{N} / \mathrm{I}$ and $\mathrm{I} / \mathrm{K}$ in the above example, going by a Medial/Lateral convention). The population properties of marginal units might be intermediates of the core stripe samples (as if their composition were a mixture) or reveal some unique features. Either way, by distinguishing marginal recording sites, the remaining core sample should be insulated from errors of stripe affiliation.

Layer identification depends upon subtler histological features. These are vitiated in Figs. 2a, 3a, and 4a by image superimposition across layers, to optimize stripes. But in Fig. 1a the entire track is visible in a single section, so layers can be discriminated. Firstly, note that the plane of section is more superficial medially (at left, where layer 3 blobs are visible in V1) than laterally (at right, where the darker staining in $\mathrm{V} 1$ reveals layer $4 \mathrm{C}$ ). In lateral V2, the external rim of the section stains more darkly than the inner part of $\mathrm{V} 2$, bordering V1, and the dark/light boundary signifies the layer $3 / 4$ border. The most lateral (rightmost) lesion is just below this border, that is, within layer 4 . The initial part of the track (leftmost) is in the more superficial part of V2 where the layer $3 / 4$ border is absent, and has been assigned to layer 3; an intermediate stretch of track has been classed as layer " 3.5 ". Layers were identified in other cases by similar means, often using additional landmarks for interpolation: for example, the cortical surface, shortly followed by the initial responsivity (layer $1 / 2$ border) and where appropriate, the point of entry into white matter, or into V1, whose layers are slightly more differentiated. Fig. 4a details this procedure, for a track that spans most layers of V2 in the lunate sulcus, having entered via white matter and layer 6 .

\section{Physiological data}

The initial goal is to test the premise that different stripes, identified histologically, are physiologically distinct: hence it is logically impermissible to use physiological characteristics as determinants of stripe borders. The graphics in Figs. $1 b-4 b$ summarize the physiological data in relation to the stripe cycle phase (and layer) determined histologically; the diffuse stripe borders are coextensive with the locations of units classified as stripe marginals, marked as vertical ticks on the baseline. Even a cursory examination reveals certain features, for example, an association of spectral sensitivity, and insensitivity to orientation, with thin stripes. The combination of data from all cases shows highly significant functional distinctions between the stripes, as in Fig. 5. Here, the number of stripe classes has been condensed from eight to five, with three pooled classes formed by the two I stripes, and the two 
(a)

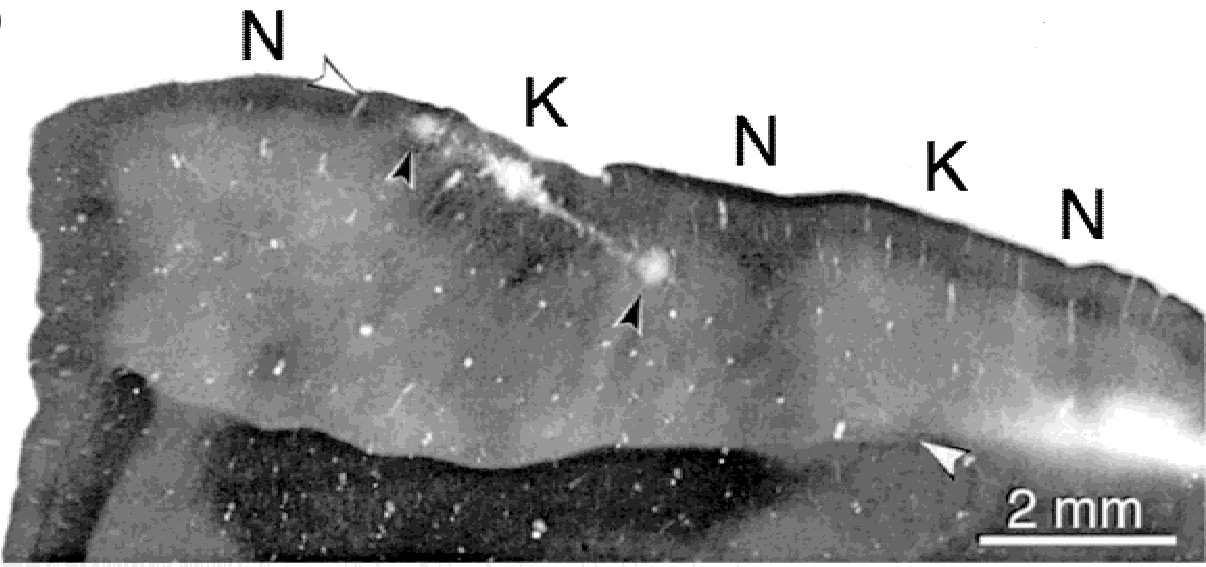

(b)

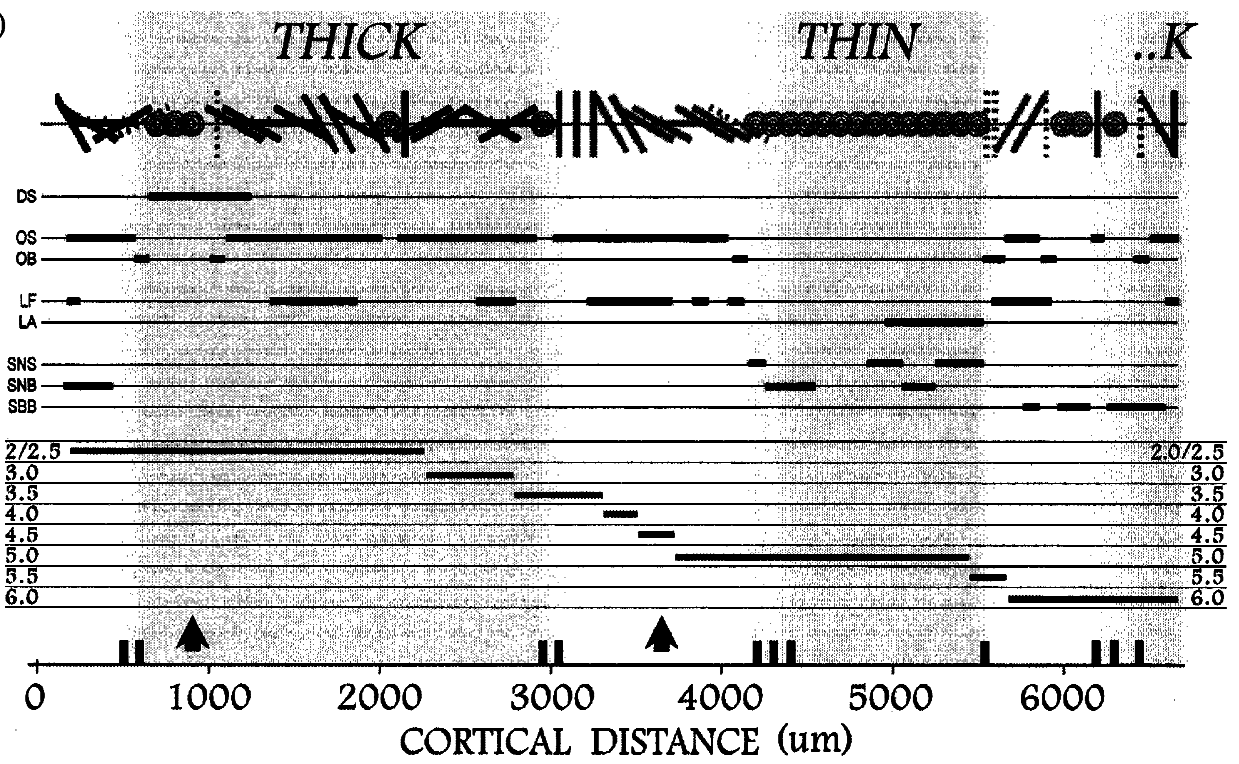

Fig. 2. (a) Electrode track with 60 units recorded across $6700 \mu \mathrm{m}$ of the external margin of V2, before entering V1 (case SP42). The first and last recorded units are marked by white arrowheads; dark arrowheads indicate track-marking lesions. The tissue incurred some local damage in the first $\mathrm{K}$ stripe during histological processing, probably an impairment of perfusion/fixation due to minor vascular disruption. The image is a composite of sections passing through layers 5 and 6 of $\mathrm{V} 2$, where the cytochrome oxidase staining is lighter, and stripes are more diffuse. The light area at right, astride the V1/V2 border, is the beginning of white matter. (b) Summary of physiological response properties, according to the conventions of Fig. 1.

pairs of marginal zones. Orientation selectivity was minimal within $\mathrm{N}$ stripes (9\%), compared to K (54\%), and I stripes $(65 \%)$. The distribution of orientation "bias" units was closer to that of nonoriented units. Directional sensitivity was found in a minority (14\%) of $\mathrm{K}$ stripe recording sites, and was absent elsewhere. Spectral sensitivity was concentrated within $\mathrm{N}$ stripes, and in the $\mathrm{N}-\mathrm{I}$ marginal zones, falling to a minimum within I stripes. There was a minor revival of spectral tuning within $\mathrm{K}$ stripes. Examining the spectral classes individually, it is the narrowband selective and bias classes, together with dark preferring units, that cluster within N stripes; the final class, "broadband bias," with weaker spectral tuning, was more frequent external to $\mathrm{N}$ stripes. Another characteristic of $\mathrm{N}$ stripes was a higher proportion (49\%) of units showing negative length (or area) summation, compared to $22 \%$ in I stripes and $33 \%$ in $\mathrm{K}$ stripes. The marginal zones had population characteristics intermediate to their neighboring stripes.

\section{Joint selectivities}

These response characteristics are not randomly assorted across recording sites. There were both positive and negative associations of particular properties, that varied between different classes of stripe. One, well-documented, dissociation is the inverse relation between orientation and spectral sensitivity; this is illustrated in Fig. 6A. The leftward trio of columns show that overall (i.e. across the whole population) the same spectral classes that cluster within $\mathrm{N}$ stripes (narrowband selective, bias and "dark" units) are dissociated from orientation tuning. However, this negative correlation is not uniform within V2 but varies across stripes, as shown in the remainder of Fig. 6A. A $G$-test was performed to quantify the 3-way (stripe-spectral-orientation) interaction, after pooling the marginal zones with $\mathrm{K}$ or $\mathrm{N}$ stripes, reducing the number of stripe classes from five to three ("K+," "N+" \& I). The outcome was heavily significant $\left(G=45.7\right.$, d.f. $\left.=8, P<10^{-6}\right)$ indicating that 
(a)


(b)

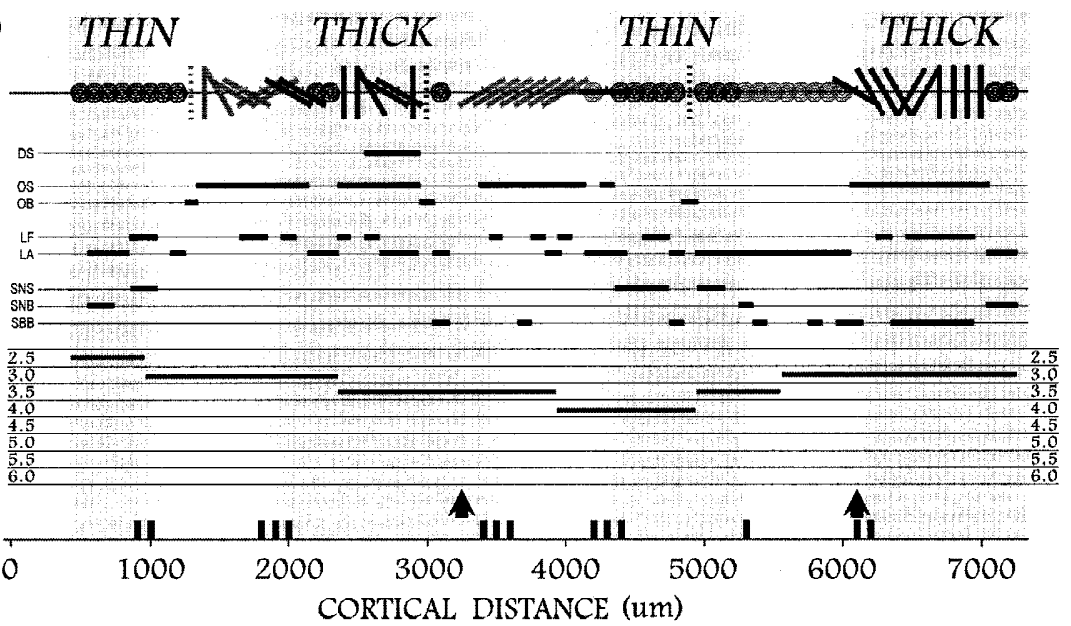

Fig. 3. (a) Electrode track with 66 units recorded across $7200 \mu \mathrm{m}$ of the convexity of V2, largely overlying white matter (case SP34). The first and last recorded units are marked by white arrowheads; dark arrowheads indicate two, track-marking lesions; the final phase of the track, lateral (rightward) to the second lesion, is not visible. The upper image is an enlarged composite of the boxed regions below. Due to slightly uneven flattening, the beginning, and end of the track with the second (more lateral) lesion are visible in one section, on the crown of the gyrus (middle boxed element), whilst the middle of the track and the first lesion appears in another section, closer to the posterior bank of the lunate sulcus (lower boxed element). (b) Summary of physiological response properties, according to the conventions of Fig. 1. When defined functionally, the medial (leftmost) thin stripe includes two units, around depth $1000 \mu \mathrm{m}$, that histologically are N/I marginals, and two further nonoriented units that, histologically, are I-stripe cells. Elsewhere, histological and functional borders are consistent. There is a small, postlesion, gap of $300 \mu \mathrm{m}$ in the recording sequence at cell 27 , depth 3100 . 
(a)
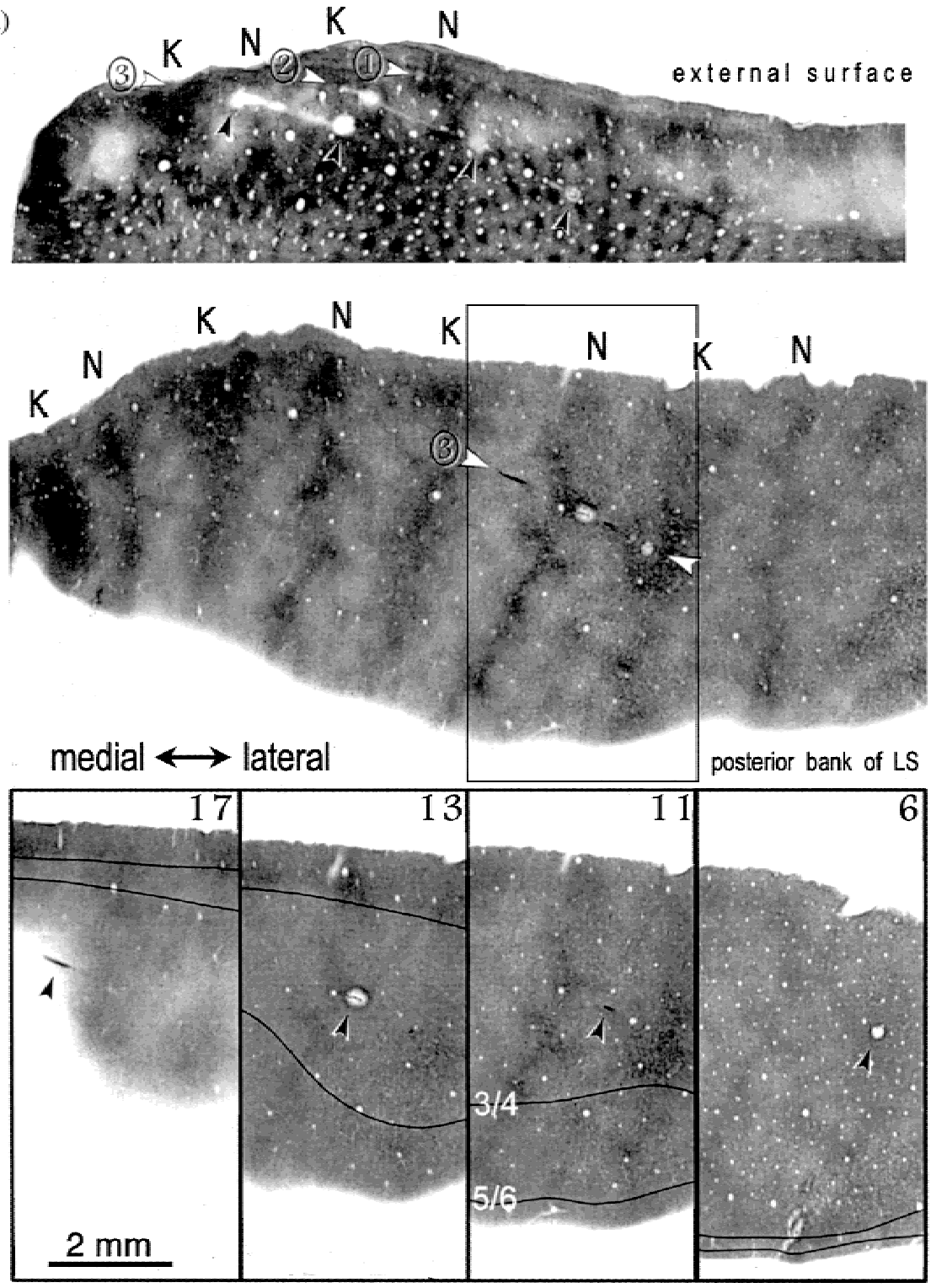

(b)

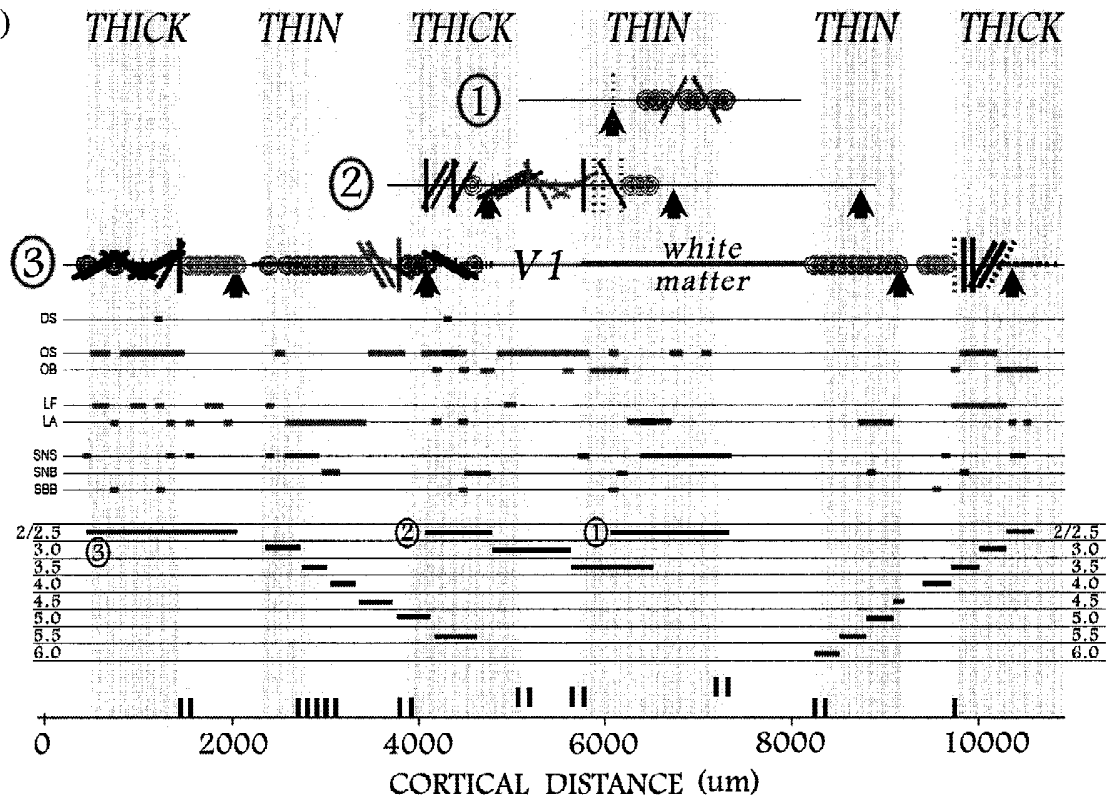

Fig. 4. (a) Three electrode tracks, with progressively more medial (leftward) entry points (case SP43). Track 1, with 10 units across $1200 \mu \mathrm{m}$ and track 2, with 24 units across $2400 \mu \mathrm{m}$ before entry into V1, were within the external margin of V2. Track 3 has 40 units across $4200 \mu \mathrm{m}$ before entering V1, crossing white matter, and recording a further 22 units in $2300 \mu \mathrm{m}$ of V2 within the lunate sulcus (LS). The top image is a composite of several sections, showing the roughly parallel courses of the electrode tracks through the external margin of V2. The first recorded units are marked by white arrowheads (coincident with a small lesion in track 1); dark arrowheads indicate other track-marking lesions. The second and third lesions in track 2 are in V1; the second lesion in track 3 is just inside V2. The middle image is a composite of sections to show the course of track 3 through the LS. The boxed area contains arrowheads indicating portions of the track that are reproduced as boxes from single sections below. The aim is to illustrate the interpolation of layers from the point of entry into layer 6 and the boundaries identified between layers $3 \& 4$, and between $5 \& 6$, traced by dark lines. Section 17-the track enters layer 6 from white matter; 13-the lesion is approximately between layers 4 and 5 (classified as " 4.5 "); 11 - the track crosses an interstripe in layer 3 , the layer where the stripes attain maximal contrast; 6-the second lesion is in layer 2, where the staining is lighter and the stripes less distinct. (b) Summary of physiological response properties, according to the conventions of Fig. 1. Physiological properties from the three tracks are superimposed on the display lines, as there are only limited regions of overlap between them. The three layer plots are separately labelled. The bars denoting histological marginal units for tracks 1 and 2 are elevated above baseline. In track 3 , there are postlesion gaps in the recording sequence of $350 \mu \mathrm{m}$ at a depth of 2050, and of $300 \mu \mathrm{m}$ at 9150 . The lateral (rightward) edge of the first $\mathrm{N}$ stripe in track 3 is relatively faint, and has a string of five units classed as N/I marginal. 



Fig. 5. Summary of stripe-related variation in sensitivity to orientation, direction, length, and spectral composition. Each histogram shows data for separate thick $(\mathrm{K})$ and thin $(\mathrm{N})$ stripes, for paired interstripes (I), and for pairs of marginal zones $(\mathrm{I} / \mathrm{K}+\mathrm{K} / \mathrm{I}=\mathrm{K}: \mathrm{I})$ and $(\mathrm{I} / \mathrm{N}+\mathrm{N} / \mathrm{I}=\mathrm{N}: \mathrm{I})$. Statistical assessment for each modality: $G$-test for random allocation of data among three physiological classes (selective, bias, unselective) and five anatomical (stripe) classes. Orientation, direction, length, and spectral composition each give $G>48$, d.f. $=8, P<10^{-7}$. NB: "spectral selective" class includes narrowband bias and dark units; length classes are positive (facilitated), unselective and negative (antagonistic plus tuned). the contingency of spectral and orientation tuning should be assessed independently in each stripe (see Table 1 for further statistical details). In $\mathrm{N}+$ stripes, the spectrally sensitive classes are clearly more frequent amongst the nonoriented population of units. The same pattern is present in $\mathrm{K}+$ stripes, yet still more accentuated; in I stripes, by contrast, the frequency of spectral sensitivity is effectively independent of orientation sensitivity. Fig. 6B shows the complementary picture, the relative frequencies of orientation tuning across spectral classes (defined by the same $G$ statistics-Table 1). Fig. 6C examines, in a similar way, the relationship between orientation and length/size tuning. Overall, there is a positive correlation of orientation tuning with length summation and, conversely, size antagonism was most frequent amongst orientation unselective units. This relationship also varies, a little, across stripes ( $G$-test for 3-way interaction: $G=19.2$, d.f. $=8, P<0.02$ ), and is weakest in $\mathrm{N}+$ stripes; $\mathrm{K}+$ stripes and I stripes are virtually identical in their length/size tuning properties. Also, there is no sign of greater levels of length antagonism (i.e. "end-stopping") among I stripe orientation sensitive units (cf. Hubel \& Livingstone 1987).

Fig. 6D examines the relationship between color and length/ size tuning, which again varies across stripes $(G=20.7$, d.f. $=8$, $P<0.01$.). There is a degree of positive coupling between color and negative size summation, which is strongest in thick stripes, weaker in thin stripes, and absent from interstripes, hence mimicking color/orientation (cf. Fig. 6B). But is the coupling of color and size a real contingency, or just a reflection of the coupling of each of these variables with orientation? The statistical analysis summarized by Table 1 shows that there is no 3-way relationship between size, spectral, and orientation sensitivity, in any of the stripes. Thus, each pairwise relationship can be assessed independent of the third variable. The strongest contingencies are between spectral and orientation sensitivity in thick and thin stripes, and between size and orientation sensitivity in thick and interstripes. Statistically, the coupling of size and spectral sensitivity is much milder, and hence the firm relationship suggested by Fig. 6D is not such a primary phenomenon.

Finally, in thick stripes, there are associations with directional sensitivity (Fig. 7A). For instance, no directional units were spectrally selective. This is significant, if other factors are ignored $(G=$ 9.6, d.f. $=1, P=0.002$ ). However, the negative coupling of direction and spectral sensitivity is not a primary phenomenon, and becomes insignificant if associations with orientation and size sensitivity are taken into account (Table 2). Importantly, for instance, directional sensitivity is positively correlated with orientation sensitivity, whereas spectral sensitivity has the reverse relationship. Spectral sensitivity is also coupled to negative size summation (as discussed above) but, from Fig. 7A, the relationship between direction and size is apparently more complex: directionselective units tend to show negative size summation but bias units do not. To test this result, spectrally selective units were excluded from subsequent analysis (because, like I and $\mathrm{N}$ stripe data, they were entirely nondirectional). Fig. 7B shows the consequent adjustment to orientation and size frequencies for the nondirectional units. There remains a mild correlation between direction and orientation, and a strong association with size (Table 2). Thus, a preference for spots (i.e. negative size/length summation) was a general characteristic of V2 direction-selective units, similar to the directional units in V1 identified to project to area V5 (Movshon $\&$ Newsome, 1996). A minority of directional units responded only to spots, being the sole directional cells classified as nonoriented. The anomalous size summation of direction-bias units is eliminated when all nonoriented units are further excluded from the data 

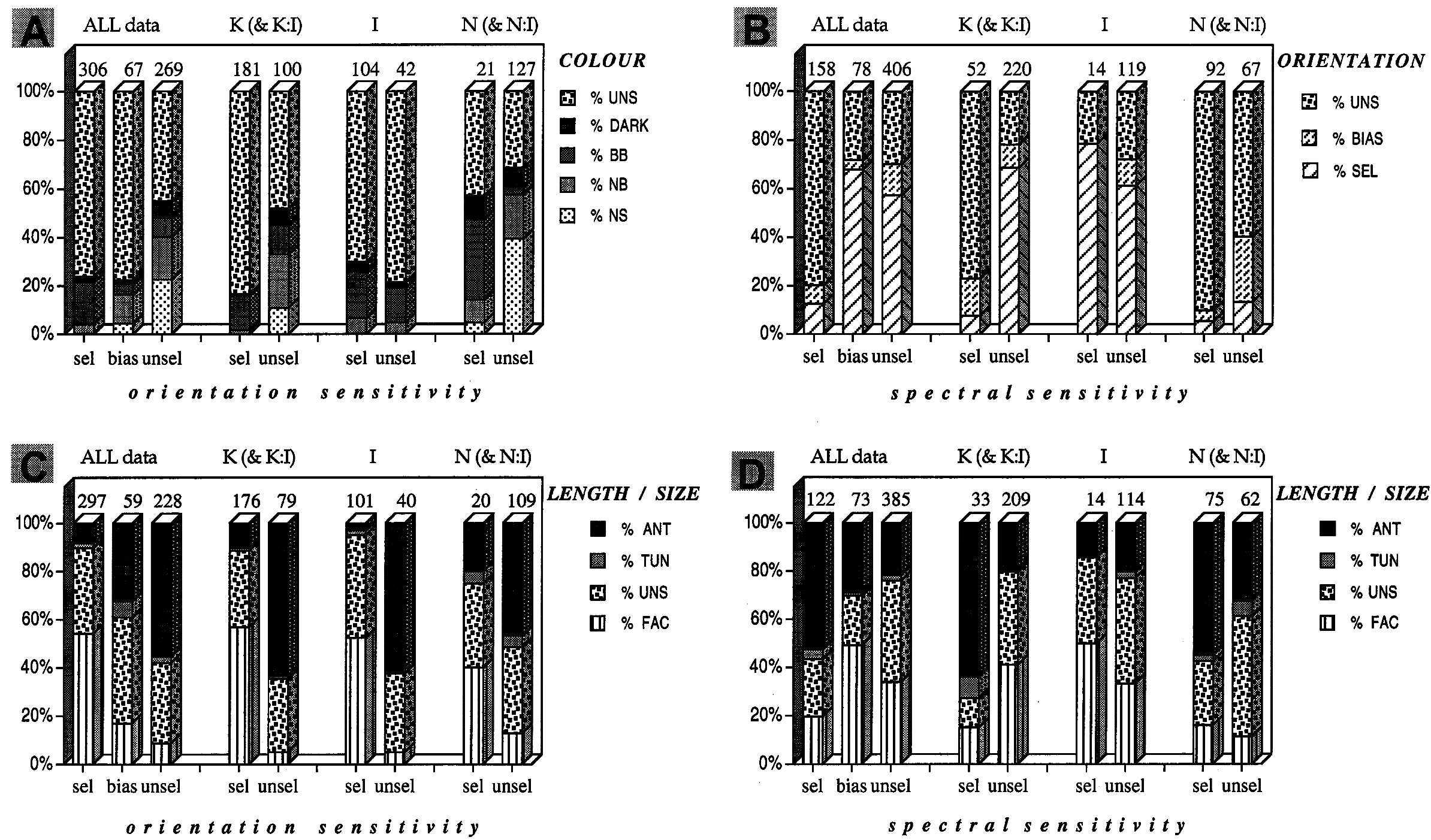

Fig. 6. Joint selectivities for orientation, spectral composition, and length/area (size), and their variation across stripes. (A) Variations in spectral sensitivity: the left trio of columns shows spectral tuning as indexed by degree of orientation selectivity; the other three pairs of columns subdivide this data (omitting orientation biased units) by stripe type. (B) Variations in orientation sensitivity: the left trio of columns shows orientation tuning as indexed by spectral sensitivity; the other three pairs of columns subdivide this data (omitting broadband biased units) by stripe type. (C) Variations in size sensitivity: the left trio of columns shows size tuning as indexed by degree of orientation selectivity; the other three pairs of columns subdivide this data (omitting orientation biased units) by stripe type. (D) Variations in size sensitivity as indexed by spectral sensitivity (conventions follow B $\&$ C). 
Table 1. Statistical assessment of association between spectral, size, and orientation sensitivity tested separately in thick stripes, thin stripes, and interstripes ${ }^{a}$

\begin{tabular}{|c|c|c|c|c|c|c|c|c|c|c|}
\hline & \multicolumn{3}{|c|}{ Thick $(\mathrm{K}+)$} & \multicolumn{3}{|c|}{ Inter $(\mathrm{I}+\mathrm{I})$} & \multicolumn{3}{|c|}{ Thin $(\mathrm{N}+)$} \\
\hline & & $G$ & $P$ & Inference & $G$ & $P$ & Inference & $G$ & $P$ & Inference \\
\hline $\begin{array}{l}3 \text {-way interaction }(\mathrm{df}=8) \\
\text { spectral } \times \text { size } \times \text { orientatior } \\
\quad(3 \times 3 \times 3 \text { levels })\end{array}$ & & 3.6 & 0.89 & nil & 8.0 & 0.44 & nil & 11.5 & 0.18 & nil \\
\hline $\begin{array}{l}\text { 2-way associations: }(\mathrm{df}=12) \\
\text { spectral } \times \text { orientation } \\
(3 \times 3 \text { levels })\end{array}$ & $\begin{array}{l}\text { at (n) levels of: } \\
\text { size } \\
(3)\end{array}$ & 30.6 & $2.3 \times 10^{-3}$ & N.C. & 16.4 & $1.7 \times 10^{-1}$ & nil & 44.7 & $1.2 \times 10^{-5}$ & N.C. \\
\hline $\begin{array}{c}\text { size } \times \text { orientation } \\
(3 \times 3 \text { levels })\end{array}$ & $\begin{array}{l}\text { spectral } \\
\text { (3) }\end{array}$ & 88.6 & $1.1 \times 10^{-13}$ & P.C. & 69.5 & $1.0 \times 10^{-8}$ & P.C. & 20.5 & $5.9 \times 10^{-2}$ & p.c. \\
\hline $\begin{array}{l}\text { spectral } \times \text { size } \\
\quad(3 \times 3 \text { levels })\end{array}$ & $\begin{array}{l}\text { orientation } \\
\text { (3) }\end{array}$ & 21.8 & $4.0 \times 10^{-2}$ & n.c. & 15.5 & $2.2 \times 10^{-1}$ & nil & 18.7 & $9.5 \times 10^{-2}$ & n.c. \\
\hline
\end{tabular}

a" $\mathrm{K}+$ " and "N+" are thick and thin stripe data pooled with their respective marginal zones. G-tests were based on three levels of each response property: selective, bias and unselective for orientation and spectral sensitivities ("spectral selective" level includes narrowband bias and dark units) and positive, neutral, or negative size summation. The initial test for a 3-way interaction is negative, for each type of stripe. It is followed by 2-way tests for the independence of each pair of stimulus variables at a given level of the third variable (i.e. abolishing any influence of the third variable on the tested pairing). The spectral $\times$ size results are the weakest, implying that the coupling seen in Fig. 7D largely depends on the mutual relationship of these stimulus variables with orientation sensitivity. df: degrees of freedom; N.C.: negative correlation; P.C.: positive correlation; and n.c.: p.c. mild negative, positive correlation.

(Fig. 7C). The change from Figs. 7B to $7 \mathrm{C}$ is a general loss of length antagonized units (which, following the strong association between length and orientation, tend to be nonoriented) and the differential effect on direction-bias units reflects a mild 3-way interaction between direction, size, and orientation specificities (Table 2). The absolute number of directional units recovered from thick stripes was not large, so it is useful to check that similar associations are evident in the total dataset (i.e. including histologically unverified directional units) shown in Figs. 7D-7E.

\section{Asymmetry within the stripe cycle}

Fig. 8a shows the distribution of orientation tuning across our full cycle parcellation (with each I stripe scored separately, plus four marginal zones). The most prominent feature of the cycle is the selectivity minimum coinciding with the $\mathrm{N}$ stripe. A secondary feature is that this minimum appears to be weighted to the lateral side of the $\mathrm{N}$ stripe: in other words, the I/N marginal zone and I stripe medial to the $\mathrm{N}$ stripe $\left(\mathrm{I}^{\mathrm{m}}\right)$ appear to be more sensitive to orientation than their equivalents situated on the lateral margin of the $\mathrm{N}$ stripe. A $2 \times 2$ contingency test (orientation selective versus unselective, and $\mathrm{I}^{\mathrm{m}}$ and $\mathrm{I} / \mathrm{N}$ versus $\mathrm{N} / \mathrm{I}$ and $\mathrm{I}^{\mathrm{l}}$ ) gives $G=$ 6.4 , d.f. $=1, P<0.02$, a result that implies a degree of functional asymmetry within the stripe cycle. Similar indications of asymmetry are present in the other modalities (Fig. 8b), since both spectrally selective and length-antagonized units are also more frequent on the lateral margins of $\mathrm{N}$ stripes: length (positive versus negative summation and $\mathrm{I}^{\mathrm{m}}$ and $\mathrm{I} / \mathrm{N}$ versus $\mathrm{N} / \mathrm{I}$ and $\left.\mathrm{I}^{\mathrm{l}}\right) G=$ 10.8 , d.f. $=1, P<0.002$; spectral (selective versus unselective and $\mathrm{I} / \mathrm{N}$ versus N/I) $G=6.3$, d.f. $=1, P<0.02$.

\section{Distribution of functional specialization across layers and stripes}

In examining the laminar distribution of stimulus response selectivity, we had two questions in mind: (1) are the response selectivities that characterize a particular type of stripe emphasized (or deemphasized) in any particular layer? (2) where a given selec- tivity is found in more than one type of stripe, is there any discernible difference in its laminar "profile" between the different stripes? Due to the manner of recording, our sample was biased toward the superficial layers, so some middle and lower layer classes have been pooled. Likewise, to maximize sample size, $\mathrm{K}$ and $\mathrm{N}$ stripes are merged with their marginal populations $(\mathrm{K}+$ and $\mathrm{N}+$ stripes). In analyzing the data, we focus on trends rather than particularize about individual layers.

A clear example of laminar specificity was provided by direction sensitivity in thick stripes, which was most frequent in the middle to upper layers, peaking in deep layer 3 (Fig. 9). Similarly, orientation selectivity was most prominent in the superficial layers of $\mathrm{K}+$ stripes and I stripes, although the laminar profiles differed slightly (Fig. 10). $\mathrm{N}+$ stripes show a lower level of orientation selectivity, with a laminar profile that was flatter overall. The particular characteristic of $\mathrm{N}+$ stripes is spectral selectivity, and this also achieves peak frequency in layer 3 (Fig. 10). In $\mathrm{K}+$ stripes, the laminar profile of spectral sensitivity is distinctly different, being U-shaped: it is minimal (10\%) in deep layer 3, rising to around $20 \%$ in the outer layers 2 and 6 (Fig. 10). I stripes, although lacking the most spectrally selective classes, also display a weighting of narrowband-bias units toward the superficial layers (Fig. 10).

Thus, a general property of these laminar profiles is for the characteristic (majority) properties of a stripe to be most prominent in the upper-middle layers. There is also a complementary trend, that the minority properties of a stripe are most common in the outer layers, for example, spectral sensitivity in $\mathrm{K}+$ stripes. It is this, symmetrical, element in laminar profiles that is again evident when examining size tuning properties. The clearest example is seen in $\mathrm{N}+$ stripes, where negative length/area summation (pooling antagonistic and tuned classes) peaks at around $60 \%$ at the layer $3 / 4$ boundary, whilst positive length summation is much less frequent and shows a complementary, basically bipolar distribution, peaking in layers $2 / 2.5$ and 6. In $\mathrm{K}+$ stripes, the overall balance of positive and negative classes is more even and both laminar profiles are essentially flat. In I stripes, the positive classes 


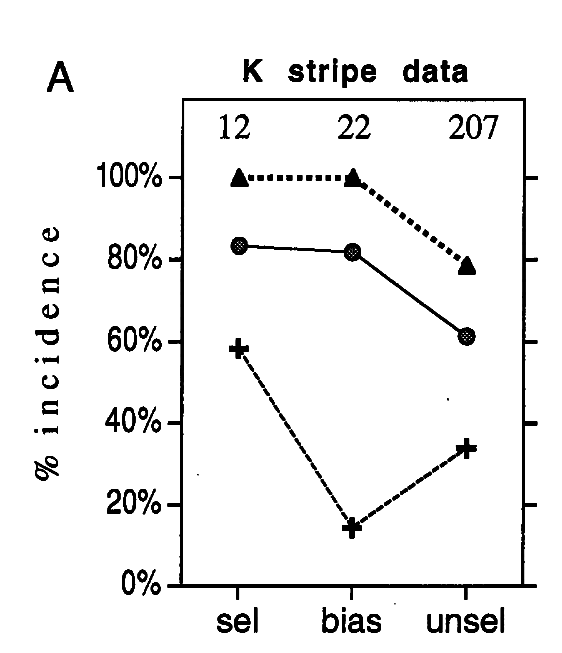

\section{B K stripe data}
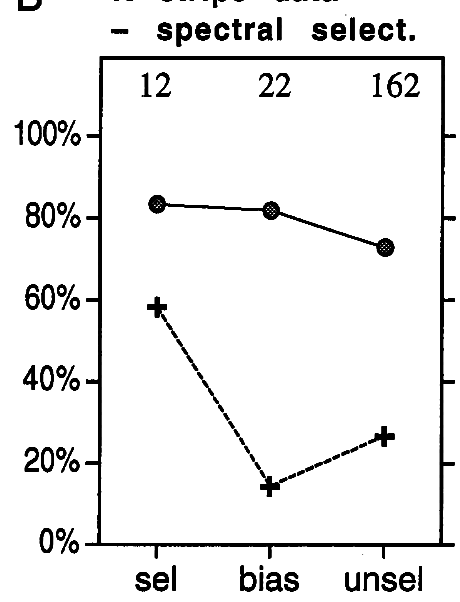

E All data

D

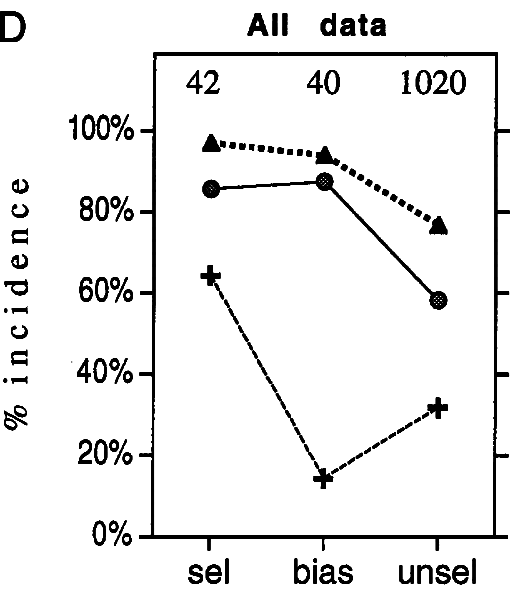

direction sensitivity

\section{- spectral select.}

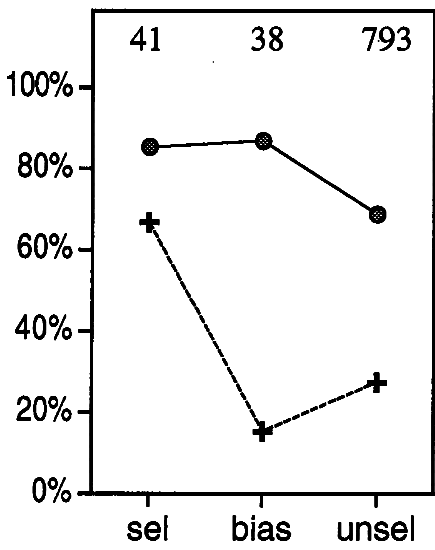

direction sensitivity
C K stripe data

- spectral select.

- orlent. unselect.

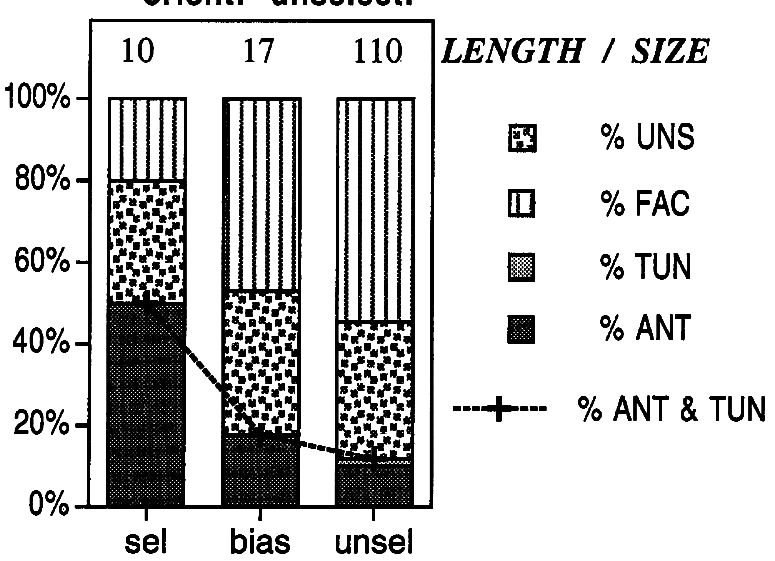

F All data

- spectral select.

- orient. unselect.

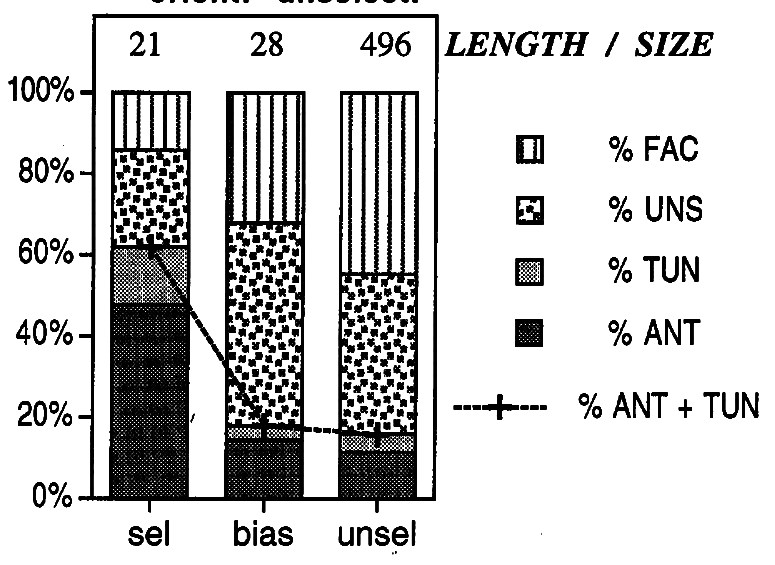

direction sensitivity 
Table 2. Statistical assessment of association of direction with spectral, size, and orientation sensitivity within thick stripes $^{a}$

\begin{tabular}{|c|c|c|c|c|}
\hline Thick stripe (K) data & & $G$ & $P$ & Inference \\
\hline $\begin{array}{l}\text { 2-way association } \\
\text { direction } \times \text { spectral } \\
\quad(2 \times 2 \text { levels })(\mathrm{df}=1)\end{array}$ & at (n) levels of: & 9.6 & 0.002 & N.C. \\
\hline $\begin{array}{l}\text { Direction } \times \text { spectral } \\
\quad(2 \times 2 \text { levels })(\mathrm{df}=4)\end{array}$ & $\begin{array}{l}\text { orientation (2) } \\
\quad \& \text { size (2) }\end{array}$ & 7.0 & 0.14 & nil \\
\hline Spectral broadband thick stripe $(\mathrm{K})$ data & & $G$ & $P$ & Inference \\
\hline $\begin{array}{l}\text { 3-way interaction }(\mathrm{df}=8) \\
\quad \text { direction } \times \text { size } \times \text { orientation } \\
\quad(3 \times 3 \times 3 \text { levels })\end{array}$ & & 15.2 & 0.06 & mild \\
\hline $\begin{array}{l}\text { 2-way associations }(\mathrm{df}=12) \\
\text { orientation } \times \text { size } \\
\quad(3 \times 3 \text { levels })\end{array}$ & $\begin{array}{l}\text { at (n) levels of: } \\
\text { direction (3) }\end{array}$ & 98.5 & $10^{-8}$ & P.C. \\
\hline $\begin{array}{l}\text { direction } \times \text { size } \\
\quad(3 \times 3 \text { levels })\end{array}$ & orientation (3) & 27.7 & 0.006 & N.C. \\
\hline $\begin{array}{l}\text { direction } \times \text { orientation } \\
\quad(3 \times 3 \text { levels })\end{array}$ & size (3) & 20.8 & 0.05 & p.c. \\
\hline
\end{tabular}

\begin{abstract}
${ }^{a}$ The first part of the table includes all thick stripe data, corresponding to Fig. 7A. The first $G$-test for direction $\times$ spectral discounts the concomitant level of orientation or size selectivity and produces a significant (negative) correlation. The second $G$-test of direction $\times$ spectral utilizes two levels of each response property: selective $\&$ bias versus unselective for direction; selective versus (broadband) bias and unselective for spectral and orientation; positive \& neutral versus negative for size. This result is not significant [-this test cannot be conducted using three levels of each property as this would imply $\mathrm{df}=36$, requiring a much larger total sample size]. The second part of the table excludes spectral selective data, and corresponds to Fig. 7B. The test for a 3-way interaction is marginal. It is followed by 2-way tests for the independence of each pair of stimulus variables at a given level of the third variable (using 3 levels of all 3 variables, with $\mathrm{df}=12$ ). The strong positive correlation (P.C.) between orientation and size means that orientation selectivity correlates with positive length summation. The negative correlation (N.C.) between direction and size means that directional selectivity correlates with negative size summation. Hence, the mild positive correlation (p.c.) between orientation and direction is not a secondary effect, for it does not reflect the opposite coupling of orientation and direction with size.
\end{abstract}

are significantly more frequent, and their distribution reaches a peak in layer 3; the minority, length antagonistic, classes have an almost flat distribution.

\section{Laminar variation of stripe distinctiveness}

The general picture arising from the complementary laminar distributions of majority and minority functional characteristics is that the stripes are most distinct from each other in the uppermiddle layers, and least distinct in the outer layers. In other words, there is a 3-way interaction between layer, stripe, and function (Table 3). We therefore proceed to examine each layer separately, using the $G$-statistic as an index of stripe distinctiveness. The outcome is shown in Fig. 11A. Each of the four tested functions attains peak distinctness in lower layer 3 . The shapes of the curves for orientation and direction sensitivity are fairly similar, dipping further in the deep layers than in the most superficial layer, such that in layer 6 the difference across stripes is insignificant. The curves for spectral selectivity and length summation each show a secondary peak in layer 5 , and roughly equal minima in layers 2 and 6. The lower histogram (Fig. 11C) shows the relative number of units tested in each layer. The sample was weighted toward the superficial layers, and this imbalance tends to boost the $G$-statistic for these layers (just as 60 heads from 100 tosses of a coin is a more significant outcome than 6 heads from 10 tosses). To control for this effect, Fig. 11B recalculates the statistical scores after normalizing the sample size across layers. Although the significance of the results in the lower and lower-middle layers is noticeably enhanced, the overall shape of the curves is not radically different and most of the conclusions reached above are still applicable. (Note that Figs. 11B and 11D are not a "truer" analysis of the results, since they depict extrapolated data; their role is to demonstrate that variation amongst layers is not due simply to uneven sample sizes).

If the translaminar variation in stripe distinctness is a simple unimodal function, it should be optimized, statistically, by pooling layers. This is demonstrated within Table 3, which compares the 6-layer interaction with two versions of a 3-layer interaction (roughly outermost $v s$. upper middle $v s$. lower middle, and uppermost $v s$. upper middle $v s$. lower); the highest score in each function is always attained by one of the latter. The curve relating to size summation (Fig. 11A) is the only one to show strong signs of being bimodal (peaking in layers 3 and 5). The notch in the curve appears to reflect the $\mathrm{N}+$ stripe data, where there is less of an imbalance of positive and negative summation in layer 4/4.5. Because of the very limited sample size, this feature of layer 4 should be treated with due caution-although it is not without a functional rationale, noted below.

\section{Discussion}

From the outset, it has seemed sensible to interpret the modular structure of area V2 as a set of specialized modules, allied to the broader picture of functionally specialized pathways coursing through primate visual cortex (DeYoe \& Van Essen, 1985, 1988; 

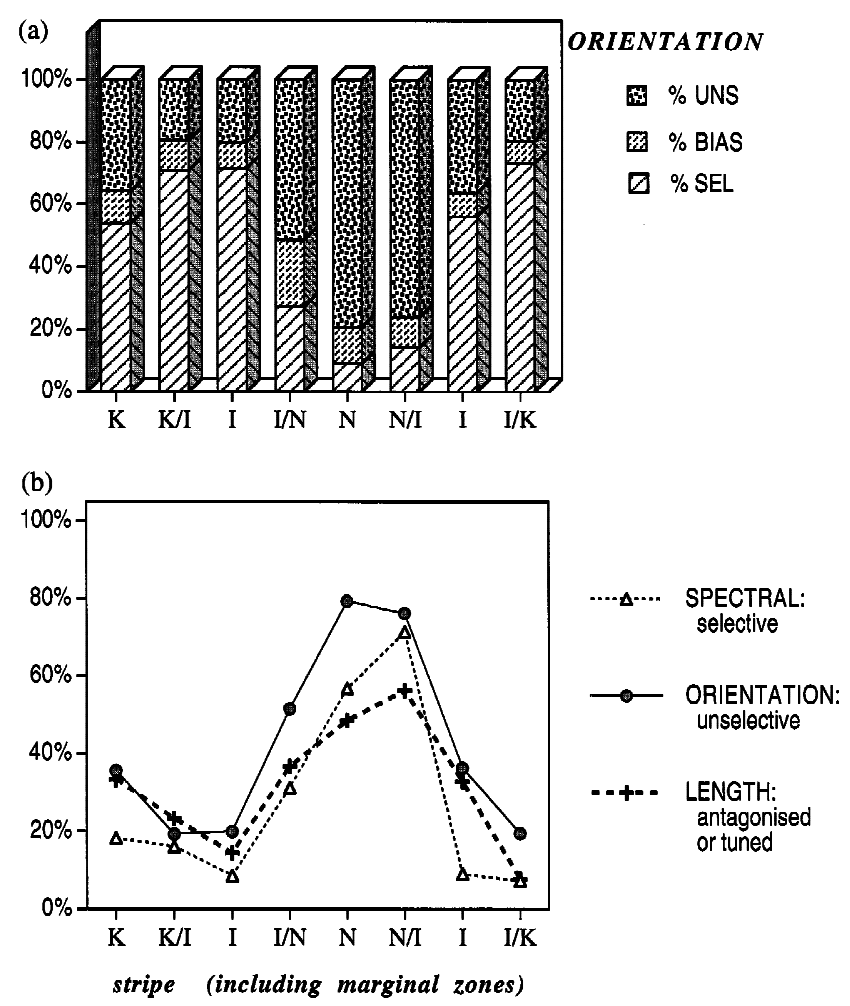

Fig. 8. Variations in functional properties across the full stripe cycle, including four single-stripe compartments and four marginal zones. (a) Orientation sensitivity. (b) Comparison of spectral selectivity, negative length summation and insensitivity to orientation. Each of these properties is characteristic of $\mathrm{N}$ stripes, but the distribution appears to be centered just lateral to the $\mathrm{N}$ stripe, as if peaking at the N/I border zone.

Hubel \& Livingstone, 1985, 1987; Shipp \& Zeki, 1985; Zeki \& Shipp, 1988). More recently, there has been a counter-trend, to deemphasize the segregation apparent in V2 whilst promoting the integrative aspects of V2 physiology (Levitt et al., 1994a,b; Gegenfurtner et al., 1996; Tamura et al., 1996; Kiper et al., 1997). Perhaps inadvertently, this has cast segregation and integration as

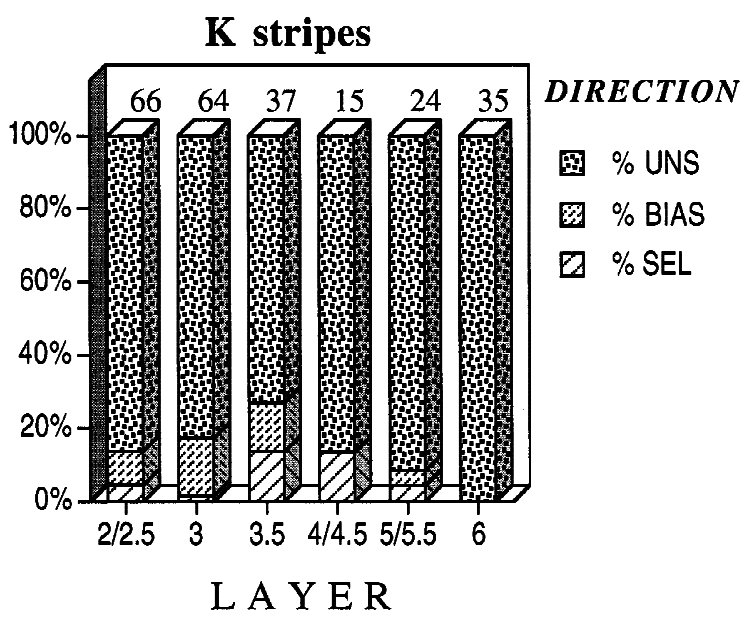

Fig. 9. The laminar distribution of directional sensitivity in K stripes. incompatible characteristics of a single area, as if one could only be performed at the cost of the other. This has not been our view (Zeki \& Shipp, 1988; Shipp \& Zeki, 1989b; Shipp, 1995); we prefer to ask how segregation and integration may be implemented as complementary operations. Our ultimate aim, in what follows, is to sketch how the present results lead to this idea, but first it is necessary to assimilate the substantial body of literature on the properties of V2 and its stripes.

\section{Segregated pathways}

In many respects, the stripes of $\mathrm{V} 2$ are a continuation of three functionally distinct compartments established in V1-blobs, interblobs, and layer 4B (Livingstone \& Hubel, 1984a; Tootell et al., 1988a,b,c; Ts'o \& Gilbert, 1988; Hubel \& Livingstone, 1990; Ts'o et al., 1990; Born \& Tootell, 1991; Edwards et al., 1995) (but see Leventhal et al., 1995, for a contrary view). The initial description of specific links from blobs to thin stripes and interblobs to interstripes (Livingstone \& Hubel, 1983) has, so far, been upheld without modification (Levitt et al., 1994b; Malach et al., 1994). Furthermore, the input to thick stripes from layer 4B, first demonstrated in squirrel monkeys (Livingstone \& Hubel, 1987), has also been found in macaques (Levitt et al., 1994b). In V1 itself, there is some degree of intrinsic cross-talk: lateral connections in layers 2 and 3 are $80 \%$ specific, in that they mostly link blob to blob, and interblob to interblob (Livingstone \& Hubel, 1984b; Yoshioka et al., 1996). Also, blobs are not watertight compartments in that the dendrites of some blob cells extend into the interblob matrix, and vice versa (Hubener \& Bolz, 1992). Finally, there are inputs from layer $4 \mathrm{~B}$ to the blobs. These form a vertical column of ascending input, but the blobs do not appear to provide reciprocal axonal output back to layer 4B (Callaway, 1998). Evidently, this degree of cross-talk does not eradicate the distinctive properties of each V1 compartment.

Within V2, by contrast, reciprocal cross-talk appears to be the rule: each type of stripe communicates with each other type of stripe, mostly within the range of a single cycle (Levitt et al., 1994b; Malach et al., 1994). Potentially, the intrinsic connections within V2 provide a substrate for crossover of functional properties between stripes, so that a 1:1 correspondence with the trio of V1 compartments cannot be taken for granted. On the other hand, there remains a clear segregation of outputs to areas like V4 and V5 (DeYoe \& Van Essen, 1985; Shipp \& Zeki, 1985, 1989b; Zeki \& Shipp, 1989b; Nakamura et al., 1993; Xiao et al., 1999), so it is not likely that functional segregation is abolished.

\section{Distinctive properties of the stripes in V2}

In the original formulation, thick stripes were characterized by orientation selectivity accompanied by disparity selectivity, interstripes by orientation selectivity with end-stopping, and thin stripes by color selectivity and minimal orientation sensitivity (Hubel \& Livingstone, 1987). Clusters of these cells were found in sequence as the electrode traversed one stripe after another, but the authors eschewed defining precise stripe boundaries, and did not tabulate the proportions of each cell class to be found in each stripe. The description was consistent with an absolute subdivision of function between stripes that subsequent workers, providing exact percentages, have found less realistic. Some retain an emphasis on specialization, whilst others stress the uniformity of stripe properties. All this work, together with the present results, is summarized in Table 4. 

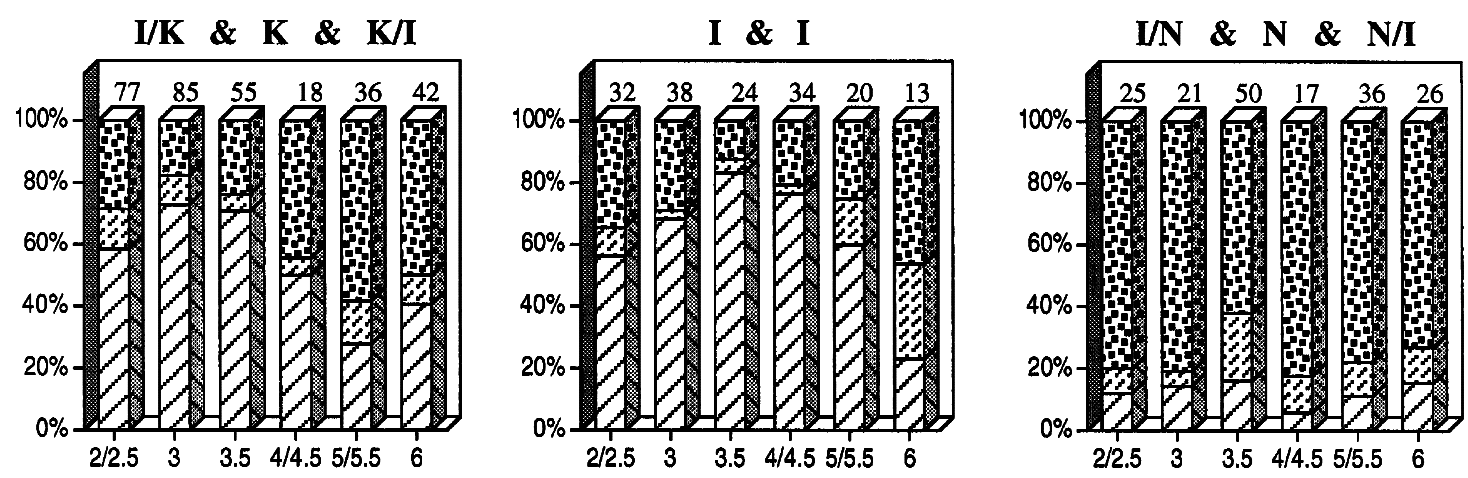

ORIENTATION
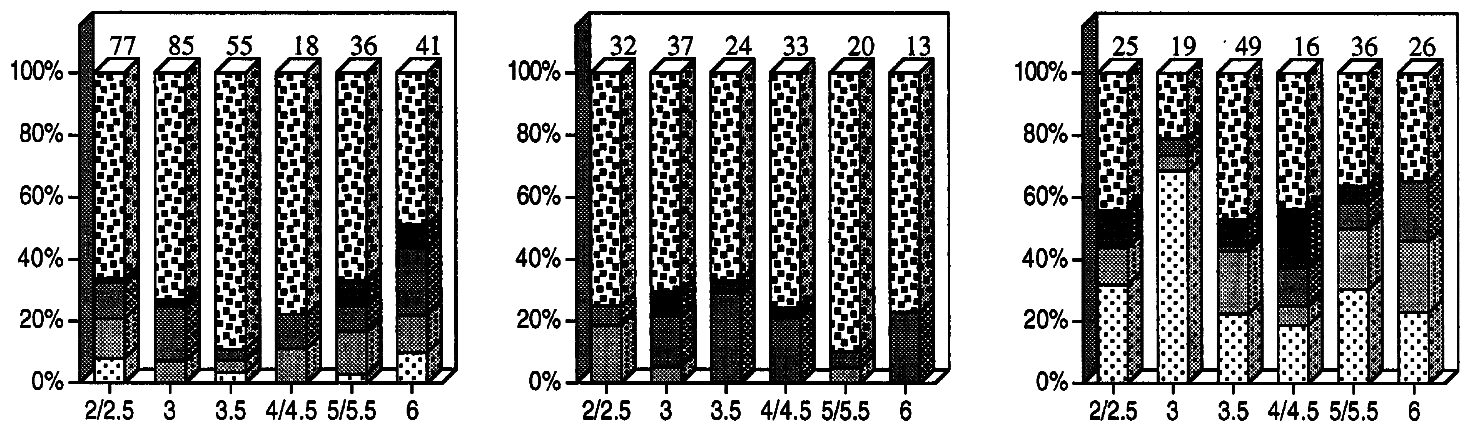

国 \% UNS

Q $\%$ BIAS

[] $\%$ SEL
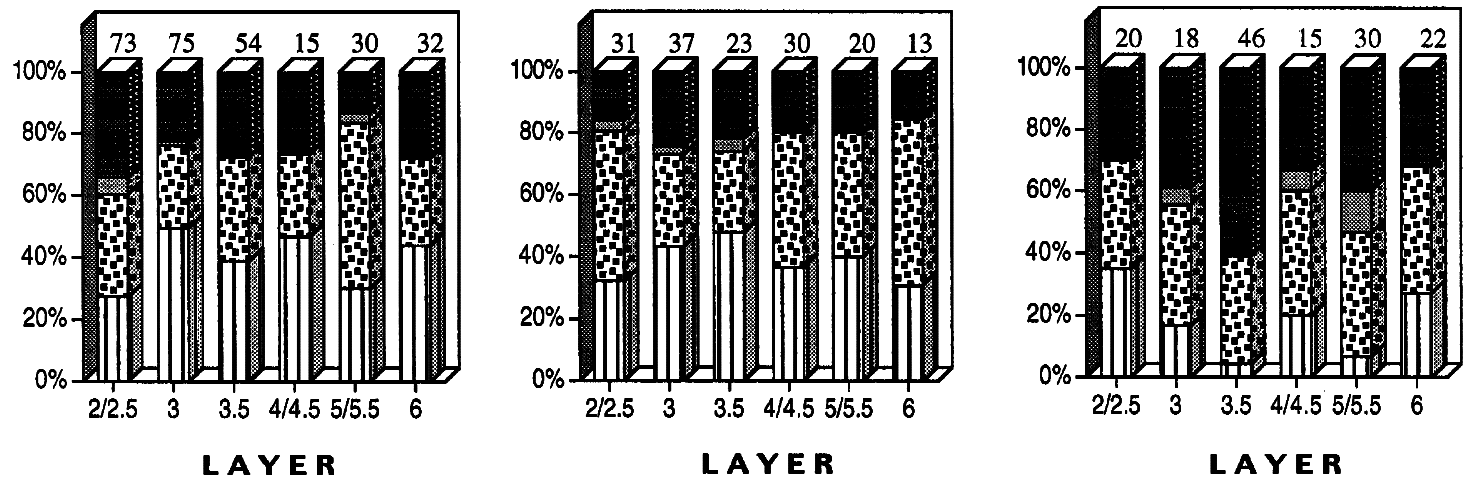

LENGTH

$\%$ ANT

$\%$ TUN

回 \% UNS

m \% FAC

Fig. 10. The laminar distribution of orientation, spectral, and length/area sensitivity in K stripes (plus marginal zones), I stripes, and $\mathrm{N}$ stripes (plus marginal zones).

Table 3. The 3-way interaction of layer, stripe, and function ${ }^{a}$

\begin{tabular}{|c|c|c|c|c|c|c|}
\hline & \multicolumn{2}{|c|}{ Orientation } & \multicolumn{2}{|c|}{ Spectral } & \multicolumn{2}{|c|}{ Size } \\
\hline & $G$ & $P$ & $G$ & $P$ & $G$ & $P$ \\
\hline \multicolumn{7}{|l|}{ 3-way interaction } \\
\hline $\begin{array}{c}\text { layer } \times \text { stripe } \times \text { function } \\
(6 \times 3 \times 3)(\text { d.f. }=20) \\
(3 \times 3 \times 3)(\text { d.f. }=8)\end{array}$ & 29.9 & 0.072 & 37.5 & 0.010 & 27.2 & 0.13 \\
\hline$(2 \& 6)$ vs. $(3 \& 3.5)$ vs. $(4 \& 4.5 \& 5 \& 5.5)$ & 16.6 & 0.034 & 18.4 & 0.018 & 21.5 & 0.0058 \\
\hline$(2 \& 3)$ vs. 3.5 vs. $(4 \& 4.5 \& 5 \& 5.5 \& 6)$ & 18.6 & 0.017 & 27.7 & 0.00053 & 11.1 & 0.19 \\
\hline
\end{tabular}

${ }^{a}$ Stripes and functional properties are considered with three levels, as before. Layers are treated with either six or three levels. The latter is generally more efficient for capturing the interaction (i. e. generates a lower $P$ value), but the optimal manner of collapsing layer categories varies with each of these three functions. 
STRIPE DISTINCTIVENESS

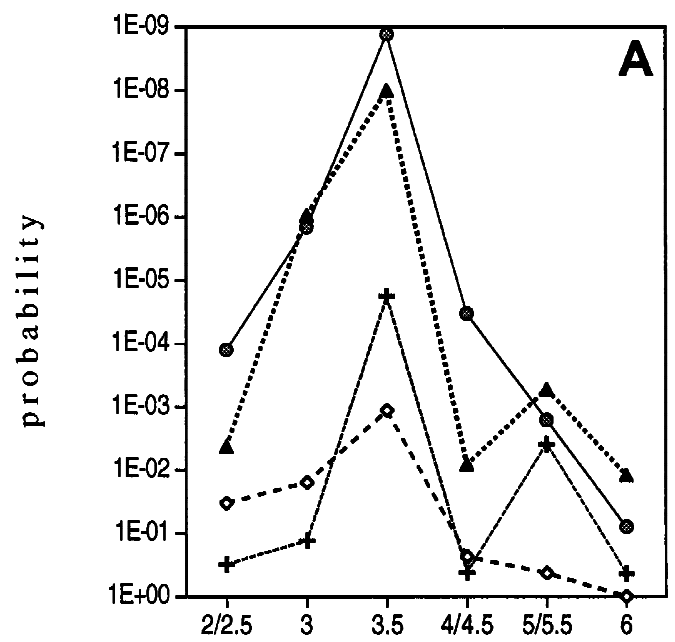

EXPERIMENTAL SAMPLES

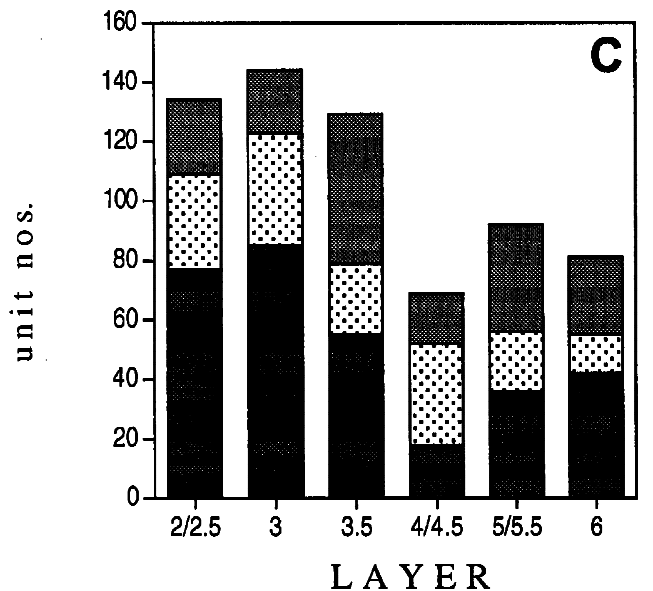

STRIPE DISTINCTIVENESS

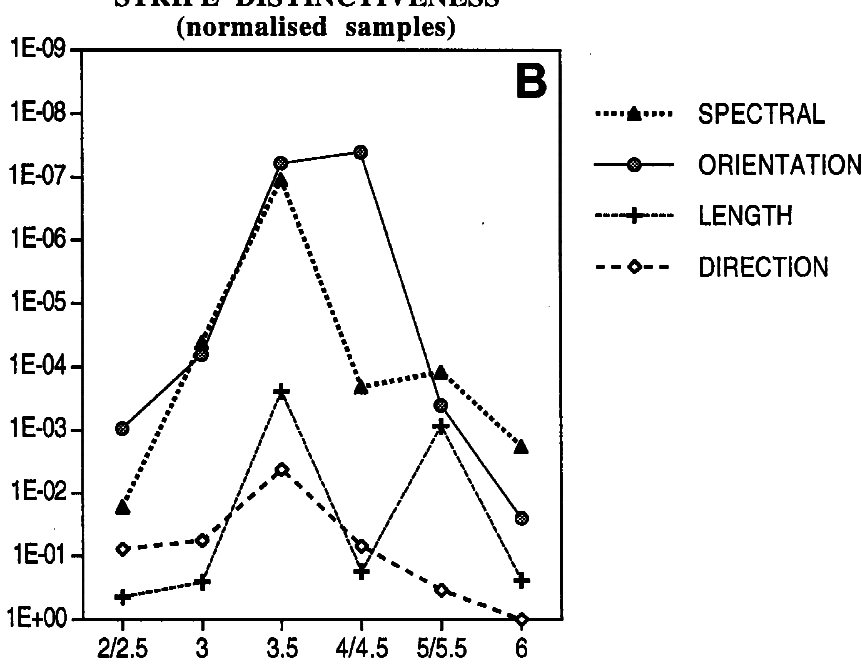

NORMALISED SAMPLES

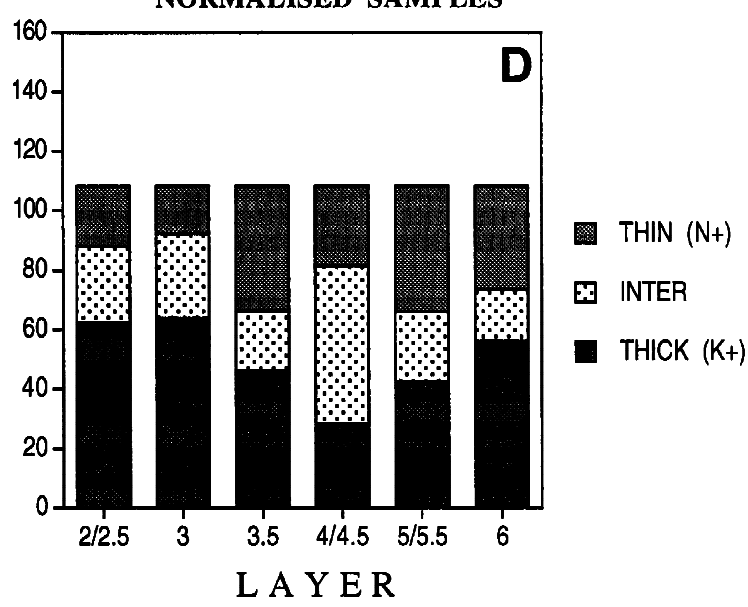

Fig. 11. The laminar variation of stripe distinctiveness, quantified by the $G$-statistic and associated $P$ value. For each layer/function, the $G$-test is $3 \times 3$ test of the 2 -way association between response class and stripe (d.f. $=4)$. Physiological classes are selective, bias, and unselective for direction, orientation, and spectral properties (spectral "selective" includes narrowband bias and dark units, as before); length classes are positive (facilitated), unselective and negative (antagonistic plus tuned). Stripe classes are $\mathrm{K}+, \mathrm{N}+$, and pooled I. The lower charts show the sample size in each compartment. Left column: statistical analysis based on actual data. Right column: analysis based on normalized data, equalizing the sample size in each layer whilst maintaining the same proportions of functional types in each compartment.

Going by the medians from Table 4, color selectivity and lesser orientation selectivity do emerge as consensus thin stripe characteristics. Notably, these thin stripe properties are also confirmed by a number of functional imaging studies (Tootell \& Hamilton, 1989; Ts'o et al., 1990; Malach et al., 1994; Roe \& Ts'o, 1995). Similarly, Table 4 confirms disparity selectivity (and possibly directional selectivity) in thick stripes, although the property of end-stopping may not be as exclusive to interstripes as originally envisaged. Median results, however, are not a very satisfactory resolution of the discrepancies that are evident across studies. There is no shortage of possible sources of these inconsistencies: variations in anesthesia, electrode characteristics/neural sampling, stimuli, criteria for response selectivity, track reconstruction and criteria for stripe identification are all potential factors. And, with relatively small populations of neurons in some studies (100 or so units), plus the known clustering of similar neurons within stripes, variability across reports of stripe properties is none too surprising. Much of the variability is thus random but some, at least, can be attributed to particular factors, histological and experimental.

\section{Stripe determination and identification}

Roe and Ts'o (1995) followed Hubel and Livingstone in using physiological criteria as the primary tool for classifying stripes. Whilst observing physiological cycles in phase with the underlying cytochrome oxidase histology, they also noted that sequences of color selective units were systematically wider than the corresponding (histological) thin stripe. Since "color" stripes were defined physiologically, it is understandable that their estimate of the relative $\%$ frequencies of color selectivity-16: 75: 12 (thick: thin: inter) - is more accentuated than any other. Our own finding, that 
Table 4. Summary of the incidence of visual selectivity across stripes, as reported by (opus):- 1. DeYoe \& Van Essen (1985); 2. Peterhans \& von der Heydt (1993); 3. Levitt et al. (1994a) [data @ Fig. 15]; 4. Roe \& Ts'o (1995) [Table 2]; 5. (Munk et al., 1995); 6. Gegenfurtner et al. (1996); 7. Tamura et al. (1996). 8. Shipp \& Zeki (2002) the present report ${ }^{a}$

\begin{tabular}{|c|c|c|c|c|c|c|c|}
\hline & Thick & Thin & Inter & Mean & $n$ & CRS & opus \\
\hline \multirow{8}{*}{$\%$ orientation selective } & 51 & 20 & 17 & 29 & 86 & o.i. $>0.7$ & 1. \\
\hline & 87 & 64 & 82 & 78 & 390 & & 2. \\
\hline & 88 & 48 & 78 & 71 & 83 & o.i. $>0.5$ & 3. \\
\hline & 86 & 38 & 84 & 69 & 426 & & 4. \\
\hline & 61 & 41 & - & 51 & 194 & & 5. \\
\hline & 85 & 73 & 96 & 85 & 100 & o.i. $>0.7$ & 6. \\
\hline & $21 *$ & $21 *$ & 38 & 27 & 55 & o.i. $>0.7$ & 7.* \\
\hline & 68 & 26 & 73 & 56 & 649 & & 8. \\
\hline Median & $77 \%$ & $40 \%$ & $78 \%$ & & & & \\
\hline \multirow[t]{7}{*}{$\%$ direction selective } & 19 & 7 & 0 & 9 & 62 & d.i. $>0.7$ & 1. \\
\hline & 30 & 21 & 34 & 28 & 190 & d.i. $>0.5$ & 2. \\
\hline & 50 & 4 & 13 & 22 & 83 & d.i. $>0.67$ & 3. \\
\hline & 60 & 3 & - & 32 & 194 & & 5. \\
\hline & 28 & 9 & 25 & 21 & 100 & d.i. $>0.7$ & 6. \\
\hline & $18^{*}$ & $18^{*}$ & 31 & 22 & 55 & d.i. $>0.7$ & 7. \\
\hline & 11 & 0 & 0 & 4 & 649 & & 8. \\
\hline Median & $28 \%$ & $7 \%$ & $19 \%$ & & & & \\
\hline \multirow{7}{*}{$\%$ color selective } & 16 & 86 & 64 & 55 & 81 & c.i. $>0.7$ & 1. \\
\hline & 10 & 27 & 27 & 21 & 111 & & 3. \\
\hline & 16 & 75 & 12 & 34 & 426 & & 4. \\
\hline & 7 & 57 & - & 32 & 194 & & 5. \\
\hline & 39 & 65 & 33 & 46 & 72 & w.i. $>1.4$ & 6. \\
\hline & $28^{*}$ & $28^{*}$ & 63 & 40 & 55 & cw.i. $>0.7$ & 7. \\
\hline & 30 & 61 & 25 & 39 & 642 & & 8. \\
\hline Median & $16 \%$ & $61 \%$ & $30 \%$ & & & & \\
\hline \multirow[t]{3}{*}{$\%$ disparity selective [1. is binocular interaction] } & 68 & 33 & 22 & 41 & 91 & bs.i. $>0.3$ & 1. \\
\hline & 38 & 21 & 15 & 25 & 390 & & 2. \\
\hline & 77 & 10 & 1 & 29 & 26 & & 4. \\
\hline Median & $68 \%$ & $21 \%$ & $15 \%$ & & & & \\
\hline \multirow{6}{*}{$\begin{array}{c}\% \text { end-stopped [8. includes length antagonism within RF] } \\
\text { 8a end-stopped and orientation sensitive } \\
\text { 8b disregards orientation sensitivity }\end{array}$} & 20 & 22 & 30 & 17 & 390 & & 2. \\
\hline & 17 & 30 & 18 & 21 & 111 & & 3. \\
\hline & 42 & 16 & 37 & 32 & 426 & & 4. \\
\hline & 13 & 19 & 41 & 24 & 76 & s.i. $>0.5$ & 6. \\
\hline & 10 & 10 & 6 & 9 & 584 & & $8 a$. \\
\hline & 29 & 47 & 22 & 33 & 584 & & $8 b$. \\
\hline Median & $20 \%$ & $22 \%$ & $30 \%$ & & & & \\
\hline
\end{tabular}

${ }^{a}$ Figures under mean are the average of entries under thick, thin, and inter; this compensates for uneven sampling across the three types of stripe, and hence the "Mean" \% data here may differ from overall \% data to be found in the original reports. *-study [7] did not distinguish between thick and thin dark stripes. [2] \& [8]: thick and thin stripe data include units classified in marginal zones; [4]: classification placed physiological criteria above histological criteria in stripe border determination; [5]: Interstripe units not included due to small sample size. CRS: criterion for response selectivity; blank entries indicate qualitative criteria; o.i: orientation index; d.i.: direction index; c.i: color index; w.i.: white index; cw.i: color/white index; bs.i.: binocular summation index; and s.i: size index.

histological thin/inter marginal zones physiologically resemble thin stripes more than interstripes, clearly echoes this fact; in Table 4, marginal zones are pooled with the proximal thin (or thick) stripe, so there is likely to be a similar effect on these color frequencies. The reason why we resort to "marginal zone" categories is that stripe borders are irregular, and cannot be localized to better than $\pm 100 \mu \mathrm{m}$ owing to the diffuse staining characteristics of cytochrome oxidase histology. All groups have to contend with this difficulty, although not all are explicit as to their resolution of the problem. On occasion, the identity of a stripe is also questionable, particularly where there are sequence irregularities. Hubel and Livingstone (1987) repeatedly commented that they found thick and thin stripes indistinguishable, in macaques, but this is to overtstate the difficulties. In our experience, the majority of banks of stripes can be sequenced with reasonable confidence (Shipp \& Zeki, 1985, 1989b; Zeki \& Shipp, 1989b). More recently, the introduction of cat 301 immunostaining has helped to certify thick stripes (DeYoe et al., 1990; Levitt et al., 1994a; Gegenfurtner 
et al., 1996). We doubt, therefore, that the data in Table 4 carry the wholesale contamination that would result from frequent stripe misidentification.

\section{Orientation and direction selectivity}

There is uniformity in the definition of these properties, but substantial variation in their estimation: for example, there is five-fold variation for orientation in interstripe data, and for direction in thick stripe data (Table 4). Stimulus variability is a weak factor, as most studies used bar stimuli, but studies [3] and [6], using sinusoidal gratings, gave higher than average yields of selectivity for orientation, but not direction (refer to the column headed "mean" showing the average incidence of selectivity pooled across stripes). The criterion for response selectivity (CRS) should be important, as shown in the context of a single study (Gegenfurtner et al., 1996) but the analysis arising from Table 4 is ambivalent. Studies [1], [6], and [7] all use the same quantitative CRS (orientation index $>0.7$ ), but together include the maximal, and minimal reported incidences of orientation sensitivity (again, refer to "mean" values). The quantitative assessments of directional sensitivity are more reassuring, namely studies [1], [3], [6], and [7] that use the same CRS (direction index >0.7), giving mostly similar "mean" frequencies, circa 20\%. In study [2] the CRS was set lower (index $>0.5$ ), and the overall incidence of direction sensitivity is closer to $30 \%$. Thus the frequency of direction-, if not orientation-, sensitive units bears some respect for the level of the CRS. In comparison to earlier results, our estimates for orientation are slightly below the median level, but well within the overall range. By contrast, our estimate of frequency for direction sensitivity is below all previous reports, and we must assume that our qualitative "CRS" was relatively severe (perhaps equivalent to "direction index $>0.9$ "). Despite these uncertainties, the choice of CRS should not greatly perturb the relative levels of stimulus specificity across stripes and, at least in this respect, there is good agreement. The general rule is that orientation sensitive units are least frequent in thin stripes and direction-sensitive units most frequent in thick stripes, with only one exception in each case.

\section{End-stopping}

Hubel and Livingstone (1987) described end-stopping as an all-or-none phenomenon, that is, one that is well assessed qualitatively; only one subsequent study [6] has actually used a quantitative CRS (size index $>0.5$ ). In Hubel and Livingstone's account, the abundance of end-stopped cells $(65 \% *)$ was the chief characteristic of the interstripes, although end-stopping could also be found in conjunction with disparity or directional selectivity, implying a presence within thick stripes, too. By definition, all end-stopped cells were also orientation selective. Subsequent studies, while testing for suppression by longer bars, have failed to specify whether the cells found to be end-stopped were also orientation selective. If the orientation criterion is relaxed, it is only to be expected that the property of end-stopping will adopt a broader distribution-as indeed reported (Table 4): only two of five studies find end-stopping most common in interstripes. The entry for our own data in Table 4 is two-fold: the first row (tagged 8a) is restricted to orientation-sensitive end-stopped cells, following the original definition, and the discrepancy with Hubel and

\footnotetext{
*See p. 3411 in Discussion of their paper. This is the only direct mention of stripe-specific \% frequency-and unfortunately it is ascribed, in a typographical error, to the thin stripes rather than the interstripes.
}

Livingstone (1987) for end-stopped cells in interstripes (at 6\% vs. $65 \%$ ) looks surprisingly large, given comparable methodology. The second row ( $8 b$ ) additionally counts as "end-stopped" a number of nonoriented cells showing negative size summation. Such cells have been named "spot cells" in previous studies (Baizer et al., 1977; Hubel \& Livingstone, 1987; Roe \& Ts'o, 1995). Likely, some (if not all) spot cells can respond to the bar stimuli used to assess end-stopping, so we have taken the second row (8b) to derive the medians in Table 4 , to correspond to the practice adopted by the other studies. $\dagger$ Our (row $8 b$ ) results are mid-range for thick stripes and interstripes. In thin stripes, we obtained higher frequencies of negative size summation, reflecting a larger contingent of nonoriented/spot cells_-a result supported by optical imaging data for V2, showing domains of size-suppression centered on thin stripes (Ghose \& Ts'o, 1997).

\section{Color selectivity}

Color selectivity has a variety of possible definitions, but the studies cited in Table 4 have all compared the response to spectrally narrowband stimuli presented with fixed luminance contrast against a dim background. Three studies employed a quantitative CRS, and these report the highest mean levels of color selectivity (refer to the "mean" column), suggesting that the qualitative CRSs used elsewhere were slightly more stringent. Despite variation in the absolute levels of color selectivity (ranging up to five-fold, in interstripes) there is a clear consensus that this level is maximal in thin stripes. The median frequencies suggest that the level of color selectivity halves from thin stripes to interstripes, and halves again from interstripes to thick stripes; looking at the studies individually, however, the vote for second place is split 50:50 between interstripes and thick stripes. Our own data place thick stripes ahead of interstripes in their level of color selectivity, in line with the results of 2-deoxyglucose imaging (Tootell \& Hamilton, 1989).

\section{Disparity selectivity and other properties}

Hubel and Livingstone's finding that disparity selectivity is most prominent in thick stripes has been confirmed by studies [1], [2], and [4] (though [1] only examined binocular summation). Study [4] (Peterhans \& von der Heydt, 1993) gave two further properties of thick stripes: responsivity to anomalous contours, and to lines defined by coherent motion. These clearly complement the general picture of thick stripe specialization in the realm of motion and dynamic form analysis. Sensitivity to these items could also be found in interstripes, but was absent from thin stripes. Levitt et al. (1994a) tested V2 cells for spatial and temporal tuning, and contrast sensitivity. The only statistically significant difference across stripes that was detected was low contrast sensitivity in thin stripes, although specific associations of (1) high contrast sensitivity with direction selectivity, and (2) spatial lowpass cells with thin stripes, were also noted. Again, these findings are in line with 2-deoxyglucose imaging that has shown preferential activation of (1) thick stripes by low contrast stimuli; (2) thick and thin stripes by low spatial-frequency stimuli, and (3) interstripes by higher spatial frequencies (Tootell \& Hamilton, 1989).

\section{Single-unit or multiunit sampling}

The choice of experimental strategy-recording at fixed intervals, or as and when a single unit is satisfactorily isolated-can cells

$†$ †xcept study [4], which specifically cites separate figures for "spot" 
exert a systematic bias over the emergent data. Multiunit data is analyzed as if the recording neighborhood is of uniform character, but any diversity amongst the recorded group of neurons would act to attenuate the measured level of selectivity. In our experiments, a single spike was recorded at a small minority of sites, but no attempt was made to secure such sites, or to verify that the resulting data was consistently single unit. Even if studies reporting single-unit data (e.g. [2], [3], [6], \& [7]) also fail to describe such procedures, the neuronal sampling was doubtless more stringent; studies [1] and [4] were more similar to ours in data composition. Table 4 shows a possible correlate for orientation and direction tuning (more prevalent in the single-unit studies) but not for color or end-stopping.

Regarding the security of our conclusions, deriving from multiunit data, it is worth noting the obvious point that diminished levels of selectivity would tend not to accentuate, but to obscure the difference between stripes, and so cannot provide a spurious basis for the conclusion that stripes are functionally specialized. In a sense, compared to single-unit data, multiunit data is a step closer to optical imaging methods that, with even lower spatial resolution, still provide clear evidence for stripe differences, for example, with respect to maps of orientation tuning in V2 (Ts'o et al., 1990; Malach et al., 1994). It is possible that some of the minority "nonoriented" multiunits recorded in thick stripes and interstripes were sited at "singularities" in the orientation map and thus, in reality, represent a composite of recordings from several different orientation-selective domains. It would be implausible, however, to suppose that the majority of nonoriented multiunits in thin stripes could have a similar origin. Similar arguments can apply, in principle, for the other functions tested.

What about joint selectivities established from multiunit recordings? In abstract terms, our analysis assumes that joint selectivity for functions $\mathrm{A}$ and $\mathrm{B}$ (i.e. $\mathrm{A}^{+} \mathrm{B}^{+}$) reflects recording from a cluster of neurons, each with this pairing of selectivities. As above, one potential concern must be that the recorded population is not uniform, but perhaps a mixture of $\mathrm{A}^{+} \mathrm{B}^{-}$and $\mathrm{A}^{-} \mathrm{B}^{+}$neurons, with $\mathrm{A}^{+} \mathrm{B}^{+}$character being an illusory pairing. Firstly, however, if this were true, the recordings could only yield apparent $\mathrm{A}^{+} \mathrm{B}^{+}$ neurons of "bias" category: the background firing of $\mathrm{A}^{-}$units accompanying $\mathrm{A}^{+}$responses to the optimum stimulus would prevent classification as $\mathrm{A}^{+}$selective (and similarly for function $\mathrm{B}$ ). Secondly, these $\mathrm{A}^{+} \mathrm{B}^{+}$"bias" neurons would be $\mathrm{A}$ or $\mathrm{B}$ in character, that is, the presence of an optimal A or B stimulus would be sufficient to stimulate greater activity. In fact, the data we recorded were typically $\mathrm{A}+\mathrm{B}+$ selective, with an "and" $\mathrm{AB}$ relationship. For example, small spot size plus best direction of motion; or small spot plus best color. One character by itself is not sufficient-for example, a small spot does not give any response from a length antagonized, directional cluster of units, if the nonpreferred direction is used. On balance, if we cannot rule out the possibility that multiunit data may reflect a heterogenous composite of neuronal selectivities, we also cannot find the grounds for serious concern. In our view, the joint selectivity data provides an additional means to characterize the differences between stripes (see below). And, even if some multiunits are heterogenous composites, the differences in local composition are still a valid means of characterizing each stripe's physiological make-up.

\section{Asymmetry within the stripe cycle?}

Table 4 shows pooled data from the two interstripes of a cycle, assuming functional equivalence. However, there are some indi- cations of dissimilarities. Roe and Ts'o (1995) found that each interstripe resembled its medial dark stripe in terms of receptivefield size and scatter; thus a thin stripe and the interstripe on its lateral border generally had a larger mean field size and scatter than a thick stripe and the following interstripe. Here we report complementary results, in that the marginal zone and interstripe on the lateral flank of a thin stripe were found to have higher levels of color sensitivity, lower levels of orientation sensitivity, and more frequent negative size summation, than the marginal zone and interstripe on the medial flank. In the companion paper, we also report that the visual representation of interstripes is mildly biased toward their medial dark stripe (Shipp \& Zeki, 2002). Individually, none of these phenomena is very telling, but together they constitute some reason to doubt that the two interstripes are entirely identical. In the extreme, one can envisage a cycle not with 3 but 4 functionally distinct components (and topographically, each half-set of interstripes has virtually complete coverage of the visual field (Shipp \& Zeki, 2002). More realistically, there could be specific partnerships between stripes, as if each interstripe were adapted to suit the functions of its medial neighbor. Clearly, the operational basis of any such functional interaction has yet to be understood. Furthermore, there is little if any anatomical evidence for such "partnerships". There is no obvious alternation in the density of connections between interstripes and area V4 (Zeki \& Shipp, 1989b; Nakamura et al., 1993; DeYoe et al., 1994; Munk et al., 1995; Felleman et al., 1997), and neither does any interstripe connect to V5 (unlike thick stripes) (Shipp \& Zeki, 1989b). Hence, the extrinsic connectivity of all interstripes resembles that of thin stripes more than thick stripes. Finally, there is no report of asymmetry in the V2-intrinsic connections of interstripes, although the relevant material might bear reexamination with this point in mind (Rockland, 1985; Cusick \& Kaas, 1988; Levitt et al., 1994b; Malach et al., 1994).

\section{Functional specificity by stripe and layer}

Extending the analysis to the third dimension of the cortex revealed that the specific functional characteristics of stripes are more pronounced in some layers than others. This new finding stems from a higher level of resolution in cortical compartmentalization, permitted by the large sample size of neurons studied. Only two studies, Levitt et al. (1994a) and Gegenfurtner et al. (1996), have previously attempted a joint stripe-by-layer analysis (using low resolution "upper," "middle," and "lower" layer classes): however, as there were only 111 or 100 units, respectively, divided amongst nine compartments ( 3 stripes $\times 3$ layers), most compartments were inadequately sampled, yielding totally inconsistent conclusions. In the current dataset, 649 units are divided amongst 72 compartments (8 stripe classes $\times 9$ layer classes). This also overstretches the data, prompting a condensation to 18 compartments ( 3 stripes $\times 6$ layers): this gives an average 36 units per compartment, a three-fold improvement on the previous reports. When analyzed in this format, the extra resolution shows that peak sensitivity is usually found in layer 3 , in either its upper or lower part; this is true for instance, of direction sensitivity in thick stripes, orientation sensitivity in thick stripes and interstripes, and for spectral sensitivity and negative size summation in thin stripes. In all these instances, we find considerably less sensitivity in layers 2 and 6. Our method of assigning a layer involved interpolation between laminar landmarks, and because of this inherent smoothing we prefer to focus on the shape of the overall laminar profile 
rather than the properties of individual layers. With a similar proviso, we note that upper and lower layer 3 (layers " 3 " and " 3.5 " in our classification) are approximations to the conventional layer 3A and layer 3B (Lund et al., 1981; Peters et al., 1997)—-terms that we have avoided up to this point, due to the absence of direct histological confirmation for this degree of laminar specificity.

In distinction to the laminar profile adopted by the majority, characteristic response properties of stripes, we also noted that some minority features (e.g. spectral sensitivity in thick stripes, positive size summation in thin stripes) had the reverse pattern, with minimal frequency in the middle layers. In other words, there is an interaction between stripe and layer location in the determination of functional properties. This means that functional differentiation across layers is best examined separately in each stripe, and that tests of layer differences made by pooling data across stripe location will tend toward insignificance (e.g. Peterhans \& von der Heydt, 1993). Here we take the other tack, and examine the variation across stripes separately in each layer. The general result is that stripes are most distinct from each other in layer 3, and least distinct in the outermost layers, that is, layers 2 and 6 (see Fig. 11A). We tested that this result was not simply due to uneven sample sizes across layers (Fig. 11B), or to the particular groupings of stripe or layer categories used in data analysis (data not shown). The laminar profiles of all response properties showed signs of a notch at layer " $4 / 4.5$ " (roughly layers $4 \& 5 \mathrm{~A}$ ), where the sample size was smallest. Once corrected for sample size, this notch only remained prominent in the curve for size summation, leaving dual peaks in layers $3 \mathrm{~B}$ and $5 \mathrm{~B}$. Effectively, this was a reflection of the raw thin stripe data, where there was much less of an imbalance between positive and negative size summation in layer 4/5A.

The fact that the stripes are most distinct from each other in layer 3 has an obvious anatomical parallel, for this is the layer that houses most of the cells projecting to higher prestriate areas, for example, from the thick stripes to V5 and from thin stripes and interstripes to V4 (Rockland \& Pandya, 1979; Lund et al., 1981; DeYoe \& Van Essen, 1985; Shipp \& Zeki, 1985). Smaller numbers of efferent cells may be found in layers 5 and 6 , or in the superficial supragranular layers, but the great majority lie in layer 3B, and there is little difference between the outputs to V4 and V5 in this respect (Shipp \& Zeki, 1989b; Zeki \& Shipp, 1989b). Layer $3 \mathrm{~B}$ of $\mathrm{V} 2$ receives some direct input from V1, although the ascending pathway is mainly focused onto layer 4 (Rockland \& Pandya, 1979; Lund et al., 1981; Weller \& Kaas, 1983; Van Essen et al., 1986). Reconstructions of individual axons show that many have terminal arbors in both layers (Rockland \& Virga, 1990). Thus the properties of V2 layer 3B cells could, in part, be inherited from their source compartments of V1: layer $4 \mathrm{~B}$ of $\mathrm{V} 1$, for instance, is known to possess relatively large numbers of direction or disparity tuned neurons (Dow, 1974; Orban et al., 1986; Hawken et al., 1988; Hubel \& Livingstone, 1990), whilst blobs and interblobs differ, like thin stripes and interstripes, in their sensitivity to orientation and spectral composition (Livingstone \& Hubel, 1984a; Tootell et al., 1988b; Ts'o \& Gilbert, 1988; Hubel \& Livingstone, 1990; Ts'o et al., 1990; Bartfeld \& Grinvald, 1992; Yoshioka \& Dow, 1996). But, very likely, there is further, local processing within layer $3 \mathrm{~B}$ - since layer 4 is often the layer with the simplest properties [e.g. V1, see Hubel \& Wiesel (1977), Blasdel \& Fitzpatrick (1984); or V5, see Lagae et al. (1989), Raiguel et al. (1995)]. One possible example in V2 concerns size summation in thin stripes where, as mentioned above, layer 4 shows a dip in the preponderance of negative size summation and in this respect resembles its input from V1 blobs, which prefer low spatial frequencies (i.e. respond well to large stimuli-Born \& Tootell, 1991; Edwards et al., 1995). The peaking frequency of certain functions in layer 3, reported here, must reflect local processing. The decline in the most superficial, or deepest layers, may reflect influences from elsewhere, as we discuss below.

\section{Integrated pathways}

It has been noted before that one rationale for the modular construction of V2-interposed, as it is, between V1 and the more singularly specialized areas of prestriate cortex-is to facilitate communication between segregated pathways, whilst permitting each to evolve semindependently (Shipp \& Zeki, 1989b; Roe \& Ts'o, 1995). Broadly, there are two distinct forms of integration that could result from exchange of information between pathways (Zeki \& Shipp, 1988): one of these is constructive, where the analysis of one attribute may assist the delineation of another (e.g. "form-from-motion"); the other is correlative, a means of registering that activity in parallel pathways is arising from the same physical object-otherwise known, more recently, as "binding" (e.g. Treisman, 1996). What evidence exists to support these conjectures?

\section{Neurons with joint selectivities}

The fact that each kind of functional property may be found in each kind of stripe can be taken to indicate that the three stripe systems do exchange signals, and are less specialized as a result (Levitt et al., 1994a,b); as reviewed above, the experimental evidence is ambivalent and partly dependent on the criteria for stripe definition. However, another pointer to mixing of signals, that is independent of stripe classification, is the fact that some neurons may be selective for more than one property, for example, color and direction, or disparity and end-stopping. Gegenfurtner et al. (1996) present a systematic analysis of selectivities for direction, orientation, color, and size, and conclude that these attributes are randomly assorted across V2 neurons, undermining stripe differences.

For comparison, Fig. 12 represents our data in the same format as Gegenfurtner et al. (1996), and includes similar results from Tamura et al. (1996). Such analysis follows the lead of Burkhalter and Van Essen (1986), who were the first to suggest that polyfunctional neurons, in ventral V2 and V3 (VP), might display random combinations of selectivities. The present data show certain nonrandom pairings of selectivities (see Figs. $6 \& 7$ )—evident, in Fig. 12, as a greater disparity in the relative height of the four bars in each group. However, it is important to note a basic similarity between our data and that of Gegenfurtner et al. (1996): in ordinal terms, the contingent frequencies amongst direction, orientation, and color are identical $\$$ (i.e. the tallest ... smallest bar in each group in Fig. 12 is the same). Thus it could be argued that the same, nonrandom contingencies are present, but attenuated in the earlier data. Take, for instance, color and motion; although the overall correlation was insignificant, Gegenfurtner et al. specifically noted that no units in their study combined a high degree of selectivity for both color and direction of motion. The negative coupling of color and direction selectivity in our data mirrors that finding (because the lesser overall frequency of these properties in

$\ddagger$ There is less similarity with regard to the property of size, defined and tested differently by the two studies. 
Tamura et al (1996)

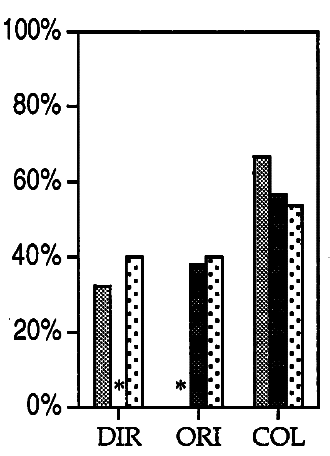

numerator function
Gegenfurtner et al (1996)

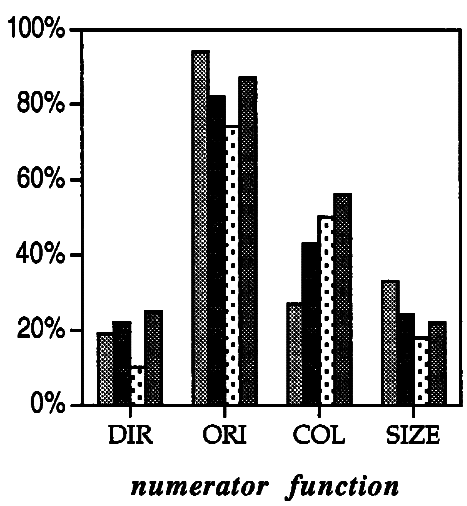

present data (full sample)

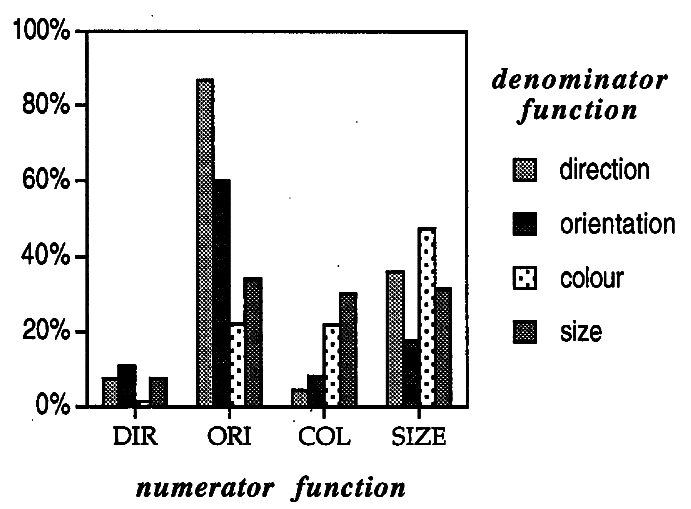

Fig. 12. Joint selectivities for stimulus attributes, as reported here and in two previous studies. The "numerator function" ( $x$ axis) is the attribute whose frequency is indicated; the "denominator function" (coded by different shadings) is the common characteristic of a subpopulation amongst which this frequency arises. Where numerator and denominator functions are the same, the frequency is that in the overall population. Thus, for example, the first block of histograms shows the incidence of directional selectivity in the whole population, followed by its incidence in the subpopulations sensitive to orientation, or color, or size. The format of the chart is taken from Gegenfurtner et al. (1996). Tamura et al. (1996) did not measure size sensitivity, and do not report joint selectivity for orientation and direction (* columns).

our dataset implies that our qualitative CRSs were relatively stringent). Taken together, the studies confirm that color and motion are not randomly assorted.

Or, take the negative coupling of orientation and color tuning. This was first reported as a mutual prohibition (Hubel \& Livingstone, 1987), as if no color-selective cell were also orientation selective. Others including Levitt et al. (1994a) and Roe and Ts'o (1995) as well as ourselves have found a negative correlation of these properties that is significant, but not absolute. Whatever the cause of this variability, it should be the same underlying relationship that appears as a weak, statistically insignificant trend in the data of Gegenfurtner et al. (1996). Our own study reveals one potential source of variability, in that the coupling of color and orientation tuning differs across stripes (see Fig. 6). Thin stripes show a clear negative correlation; in interstripes, the incidence of spectral sensitivity is equal amongst oriented and nonoriented units (random assortment); and in thick stripes the negative correlation exceeds that of thin stripes, such that here the mutual prohibition is almost absolute. ${ }^{\S}$ In general, therefore, although we do not believe that the "hallmark" properties of stripes are randomly assorted, it is equally clear that these properties are not absolutely segregated. The pattern of their interrelationships is relevant to the functions of each of the three stripe systems, as well as to the function of the striped architecture itself.

\section{Constructive integration and cue-invariance}

An implication of "cue-invariance" is that there is no simple, 1:1 relationship between the perceptual attribute served by a pathway, and its component cellular properties. For instance, in thin stripes chromatic signals may subserve color vision per se (encoding surface reflectance properties) whereas outside thin stripes such signals may be coding surface contours, depths, and directions of motion. The parvocellular inputs that deliver chro-

${ }^{\S}$ Note also that a negative coupling of color and orientation equates to $\mathrm{A}^{+} \mathrm{B}^{-}$(in the abstract formalism of the previous section)-and it is implausible that $\mathrm{A}^{+} \mathrm{B}^{-}$could arise as a spurious multiunit composite from a local population of $\mathrm{A}^{+} \mathrm{B}^{+}$and $\mathrm{A}^{-} \mathrm{B}^{-}$. matic signals to $\mathrm{V} 1$ are almost certainly mixed with other channels by the intrinsic circuitry operating within layers $4 \mathrm{~A}, 3$, and 2 (Lachica et al., 1992; Nealey \& Maunsell, 1994; Yoshioka et al., 1994; Callaway, 1998), and the intrinsic circuitry of V2 can effect further mixing. In human psychophysics, there is ample evidence that chromatic signals act as a significant supplement to luminance signals for the discrimination of speed and direction of motion (Lee \& Stromeyer, 1989; Cavanagh \& Anstis, 1991; Gegenfurtner \& Hawken, 1995; Stromeyer et al., 1995; Cropper \& Derrington, 1996; Cavanagh et al., 1998; Dougherty et al., 1999; Croner \& Albright, 1999). A contribution of color to form discrimination is equally likely, if not so well documented. Thus, the combination of color sensitivity with orientation, size, or direction sensitivity in $\mathrm{V} 2$ is potentially indicative of a system harnessing chromatic contrast for seeing form and motion. It is not immediately obvious, moreover, that units performing this role should display overt color tuning, that is, retain sensitivity to the sign of chromatic contrast. For example, some complex, orientation-selective, interblob cells of layers $2 / 3$ in $\mathrm{V} 1$ have been reported to retain responsivity to contours formed by pure chromatic contrast (i.e. isoluminant stimuli) without being color selective (Gouras \& Kruger, 1979; Thorell et al., 1984; Hubel \& Livingstone, 1990). An equivalent form of responsivity has been recorded from complex cells of V2, that are tuned to a particular axis in a 3D color space whilst being insensitive to the phase of the stimulus (Kiper et al., 1997). Cells of this class are also responsive to luminance, expressing spatiotemporal sensitivity that utilizes both forms of contrast (Johnson et al., 2001).

Note, for a converse, that oriented cells could conceivably contribute to color vision per se, since perceived color is known to fill-in from perceived boundaries (Krauskopf, 1963; Ware \& Cowan, 1983; Purves et al., 1999). The integration of form and color pathways proceeds over several levels, since both thin stripes and interstripes project on to areas V4 and TEO, terminating in further complex modules (Zeki \& Shipp, 1989b; Nakamura et al., 1993; DeYoe et al., 1994; Felleman et al., 1997; Xiao et al., 1999). However, the asymmetric relationships of color and orientation selectivity at the level of V2 - negatively correlated in thin stripes, 
but randomly assorted in interstripes - might indicate asymmetric stages, or modes, of integration, in the form and color pathways. For comparison, a high negative correlation between color and direction/orientation was also a feature of thick stripes. This may be taken as an involvement with color per se and, if so, points to something other than constructive integration.

\section{Correlative integration}

Our hypothesis for the role of color cells in thick stripes is that they are a constituent of the neural circuitry that achieves correlated firing of cells responding to different attributes of the same physical stimulus, say its color and motion properties. Clearly, we cannot directly substantiate this extension to the theory of feature integration (Singer \& Gray, 1995; Engel et al., 1997); the aim, here, is just to show that it is not antithetical to the picture of modular specialization in $\mathrm{V} 2$.

About one in five cells in thick stripes were spectrally selective, and they were concentrated toward the outer layers (2 and 6). These layers make very little contribution to the forward projections of V2, but they are the source of feedback to V1 (Lund et al., 1981; Kennedy \& Bullier, 1985; Rockland, 1994), and the target of feedback from V4 and TEO (Rockland et al., 1994). Furthermore, they participate in intrinsic connections. Cells in layers $2 / 3 \mathrm{~A}$, for instance, receive input from layer 3B (where directional, forwardprojecting cells are concentrated) and contribute their own axon collaterals to the horizontal fiber plexus that courses through layer 3B (Lund et al., 1981). Anatomically, therefore, the outer layers are primarily implicated in integrative processes. None of the spectrally selective, thick stripes units were sensitive to our test of directionality. Where cells do display joint selectivity for color and motion, it seems that the presence of either preferred feature is sufficient (Gegenfurtner et al., 1996; Tamura et al., 1996); for instance, Tamura et al. (1996) illustrate a unit that responds to either an achromatic light slit moving rightwards, or a static blue spot. Notably, the cell does not require a combination of these features, as it should if it were coding for a rightward moving, light blue object. Given these OR (as opposed to AND) integrative characteristics, and the laminar location of spectral cells in thick stripes, there is little reason to suppose that their role is to code feature conjunctions for direct interpretation by higher areas.

As an alternative, we propose that spectrally selective cells in thick stripes are a kind of "bridge", promoting synchronization between highly directional cells in layer $3 \mathrm{~B}$ of thick stripes, and spectral cells in layer 3B of thin stripes. The functional potential of such synchronization has been explored elsewhere (Tononi et al., 1992; Schillen \& Konig, 1994; Singer \& Gray, 1995; Engel et al., 1997; Singer, 1998, 1999; Gray, 1999) with attendant difficulties noted (Ghose \& Maunsell, 1999; Shadlen \& Movshon, 1999). The hypothesis calls for synchronization over the range of at least one cycle; it is known, even in anesthetized animals, that synchronization can be seen in cell pairs sited in separate stripes, up to $2 \mathrm{~mm}$ apart, and is also more frequent in V2 than V1 (Tamura et al., 1996). By symmetry, we should also predict the existence of directional selectivity amongst the upper and lower layers of thin stripes (and interstripes) - perhaps a lesser degree of selectivity that escaped our own tests, if not all others. We anticipate that the primary source of such "atypical" forms of selectivity in the outer layers is feedback from higher prestriate areas. Feedback from both V4 and V5, for instance, is known to spread diffusely within layers 1 and 6 of V2, invading all three stripe systems (Krubitzer \& Kaas, 1989; Shipp \& Zeki, 1989b; Zeki \& Shipp, 1989b). Many elements of intrinsic circuitry, including widespread lateral connections (the fiber plexus in 3B), and more local inhibitory circuitry (Kritzer et al., 1992), could promote and maintain synchronization over the long term of the network's activity (Bush \& Sejnowski, 1996; Neltner et al., 2000).

In summary, knowing the 3D compartmental organization of $\mathrm{V} 2$, it is now possible to envisage how segregation and integration are implemented as complementary functional processes. The ascending pathways through V2 are clearly segregated, both in their input and output, and occupy the internal layers. Cross-talk between these pathways does not necessarily blur this segregation, or diminish their relative specialization, as previously mooted (Levitt et al., 1994a,b; Gegenfurtner et al., 1996) . Rather, cross-links can facilitate each pathway in cue-invariant coding for selective features, and they can foster feature integration by acting to coordinate the pathways' separate activities. These aspects of function are not necessarily unique to V2 as an area, but are perhaps made more accessible by the boldness of its anatomical structure.

\section{Acknowledgments}

We thank Ian Wilson for technical assistance. This work was supported by the Wellcome Trust, UK.

\section{References}

AlbRight, T.D. (1992). Form-cue invariant motion processing in primate visual cortex. Science 255, 1141-1143.

BAizer, J.S., Robinson, D.L. \& Dow, B.M. (1977). Visual responses of area 18 neurons in awake, behaving monkey. Journal of Neurophysiology 40, 1024-1037.

Bartfeld, E. \& Grinvald, A. (1992). Relationships between orientationpreference pinwheels, cytochrome oxidase blobs, and ocular-dominance columns in primate striate cortex. Proceedings of the National Academy of Sciences of the U.S.A. 89, 11905-11909.

Benevento, L.A. \& RezaK, M. (1976). The cortical projections of the inferior pulvinar and adjacent lateral pulvinar in the rhesus monkey (Macaca mulatta): An autoradiographic study. Brain Research 108 $1-24$.

Blasdel, G.G. \& FitzPatrick, D. (1984). Physiological organization of layer 4 in macaque striate cortex. Journal of Neuroscience 4, 880-895.

Born, R.T. \& ToOTELl, R.B.H. (1991). Spatial frequency tuning of units in macaque supragranular striate cortex. Proceedings of the National Academy of Sciences of the U.S.A. 88, 7066-7070.

Burkhalter, A. \& VAN Essen, D.C. (1986). Processing of color, form and disparity information in visual areas VP and V2 of ventral extrastriate cortex in the macaque monkey. Journal of Neuroscience 6, 2237-2351.

Bush, P. \& SEJnowski, T. (1996). Inhibition synchronizes sparsely connected cortical neurons within and between columns in realistic network models. Journal of Computational Neuroscience 3, 91-110.

Callaway, E.M. (1998). Local circuits in primary visual cortex of the macaque monkey. Annual Review of Neuroscience 21, 47-74.

Cavanagh, P. \& Anstis, S. (1991). The contribution of color to motion in normal and color-deficient observers. Vision Research 31, 2109-2148.

Cavanagh, P., Henaff, M.A., Michel, F., Landis, T., Troscianko, T. \& INTRILIGATOR, J. (1998). Complete sparing of high-contrast color input to motion perception in cortical color blindness. Nature Neuroscience 1, 242-247.

Croner, L.J. \& Albright, T.D. (1999). Seeing the big picture: integration of image cues in the primate visual system. Neuron 24, 777-789.

Cropper, S.J. \& Derrington, A.M. (1996). Rapid color-specific detection of motion in human vision. Nature 379, 72-74.

Curcio, C.A. \& HARTING, J.K. (1978). Organization of pulvinar afferents to area 18 in the squirrel monkey: evidence for stripes. Brain Research 143, 155-161.

Cusick, C.G. \& KaAs, J.H. (1988). Cortical connections of area 18 and dorsolateral visual cortex in squirrel monkeys. Visual Neuroscience 1, 211-237.

Derrington, A.M., Krauskopf, J. \& Lennie, P. (1984). Chromatic mechanisms in lateral geniculate nucleus of macaque. Journal of Physiology 357, 241-265. 
De Valois, R.L., Cottaris, N.P., Elfar, S.D., Mahon, L.E. \& Wilson, J.A. (2000). Some transformations of color information from lateral geniculate nucleus to striate cortex. Proceedings of the National Academy of Sciences of the U.S.A. 97, 4997-5002.

DeYoe, E.A., Hockfield, S., Garren, H. \& Van Essen, D.C. (1990). Antibody labelling of functional subdivisions in visual cortex: cat-301 immunoreactivity in striate and extrastriate cortex of the macaque monkey. Visual Neuroscience 5, 67-81.

DeYoe, E.A., Felleman, D.J., Van Essen, D.C. \& McClendon, E. (1994). Multiple processing streams in occipitotemporal visual cortex. Nature 371, 151-154.

DeYoe, E.A. \& VAn Essen, D.C. (1985). Segregation of efferent connections and receptive field properties in visual area 2 of the macaque. Nature 317, 58-61.

DeYoe, E.A. \& VAn Essen, D.C. (1988). Concurrent processing streams in monkey visual cortex. Trends in Neuroscience 11, 219-226.

Dougherty, R.F., Press, W.A. \& Wandell, B.A. (1999). Perceived speed of colored stimuli. Neuron 24, 893-899.

Dow, B.M. (1974). Functional classes of cells and their laminar distribution in monkey visual cortex. Journal of Neurophysiology 37, 927-946.

Edwards, D.P., Purpura, K.P. \& KaPlan, E. (1995). Contrast sensitivity and spatial frequency response of primate cortical neurons in and around the cytochrome oxidase blobs. Vision Research 35, 1501-1523.

Engel, A.K., Roelfsema, P.R., Fries, P., Brecht, M. \& Singer, W. (1997). Role of the temporal domain for response selection and perceptual binding. Cerebral Cortex 7, 571-582.

Felleman, D.J. \& Van Essen, D.C. (1991). Distributed hierarchical processing in the primate cerebral cortex. Cerebral Cortex 1, 1-47.

Felleman, D.J., Xiao, Y. \& McClendon, E. (1997). Modular organization of occipito-temporal pathways: cortical connections between visual area 4 and visual area 2 and posterior inferotemporal ventral area in macaque monkeys. Journal of Neuroscience 17, 3185-3200.

Gegenfurtner, K.R. \& Hawken, M.J. (1995). Temporal and chromatic properties of motion mechanisms. Vision Research 35, 1547-1563.

Gegenfurtner, K.R., Kiper, D.C. \& Fenstemaker, S.B. (1996). Processing of color, form and motion in macaque area V2. Visual Neuroscience 13, 161-172.

Ghose, G.M. \& Maunsell, J. (1999). Specialized representations in visual cortex: a role for binding? Neuron $\mathbf{2 4}, 79-85,111-125$.

Ghose, G.M. \& Ts'o, D.Y. (1997). Form processing modules in primate area V4. Journal of Neurophysiology 77, 2191-2196.

Gouras, P. \& KRUGER, J. (1979). Responses of cells in foveal visual cortex of the monkey to pure color contrast. Journal of Neurophysiology 42, $850-860$.

Gray, C.M. (1999). The temporal correlation hypothesis of visual feature integration: still alive and well. Neuron 24, 31-47, 111-125.

HaWken, M.J., PARKer, A.J. \& Lund, J.S. (1988). Laminar organization and contrast selectivity of direction selective cells in the striate cortex of the Old-World monkey. Journal of Neuroscience 8, 3541-3548.

Hubel, D.H. \& Wiesel, T.N. (1977). Ferrier lecture. Functional architecture of macaque monkey visual cortex. Proceedings of the Royal Society $B$ (London) 198, 1-59.

Hubel, D.H. \& Livingstone, M.S. (1985). Complex-unoriented cells in a subregion of primate area 18 . Nature $\mathbf{3 1 5}, 325-327$.

Hubel, D.H. \& Livingstone, M.S. (1987). Segregation of form, color and stereopsis in primate area 18. Journal of Neuroscience 7, 3378-3415.

Hubel, D.H. \& Livingstone, M.S. (1990). Color and contrast sensitivity in the lateral geniculate body and primary visual cortex of the macaque monkey. Journal of Neuroscience 10, 2223-2237.

Hubener, M. \& Bolz, J. (1992). Relationships between dendritic morphology and cytochrome oxidase compartments in monkey striate cortex. Journal of Comparative Neurology 324, 67-80.

Johnson, E.N., Hawken, M.J. \& Shapley, R. (2001). The spatial transformation of color in the primary visual cortex of the macaque monkey. Nature Neuroscience 4, 409-416.

Kennedy, H. \& Bullier, J. (1985). A double-labelling investigation of the afferent connectivity to cortical areas $\mathrm{V} 1$ and V2 of the macaque monkey. Journal of Neuroscience 5, 2815-2830.

Kiper, D.C., Fenstemaker, S.B. \& Gegenfurtner, K.R. (1997). Chromatic properties of neurons in macaque area V2. Visual Neuroscience 14, 1061-1072.

KRAUSKOPF, J. (1963). Effect of retinal image stabilization on the appearance of heterochromatic targets. Journal of the Optical Society of America 53, 741-744.

Kritzer, M.F., Cowey, A. \& Somogyi, P. (1992). Patterns of inter- and intralaminar GABAergic connections distinguish striate (V1) and extrastriate (V2, V4) visual cortices and their functionally specialized subdivisions in the rhesus monkey. Journal of Neuroscience 12, 4545-4564.

Krubitzer, L.A. \& KaAs, J.H. (1989). Cortical integration of parallel pathways in the visual system of primates. Brain Research 478, 161-165.

Lachica, E.A., Beck, P. \& Casagrande, V.A. (1992). Parallel pathways in macaque monkey striate cortex: Anatomically defined columns in layer III. Proceedings of the National Academy of Sciences of the U.S.A. 89, 3566-3570.

Lagae, L., Gulyas, B., Raiguel, S. \& Orban, G.A. (1989). Laminar analysis of motion information processing in macaque V5. Brain Research 496, 361-367.

Lee, J. \& Stromeyer, C.F.D. (1989). Contribution of human short-wave cones to luminance and motion detection. Journal of Physiology 413, 563-593.

Lennie, P., Krauskopf, J. \& Sclar, G. (1990). Chromatic mechanisms in striate cortex of macaque. Journal of Neuroscience 10, 649-669.

Leventhal, A.G., Thompson, K.G., Liu, D., Zhou, Y. \& Ault, S.J. (1995). Concomitant sensitivity to orientation, direction, and color of cells in layers 2, 3 and 4 of monkey striate cortex. Journal of Neuroscience 15, 1808-1818.

LevitT, J.B., KiPer, D.C. \& Movshon, J.A. (1994a). Receptive fields and functional architecture of macaque V2. Journal of Neurophysiology 71, 2517-2542.

Levitt, J.B., Yoshioka, T. \& LUND, J.S. (1994b). Intrinsic cortical connections in macaque visual area V2: evidence for interaction between different functional streams. Journal of Comparative Neurology 342, 551-570.

Levitt, J.B., Yoshioka, T. \& Lund, J.S. (1995). Connections between the pulvinar complex and cytochrome oxidase-defined compartments in visual area V2 of macaque monkey. Experimental Brain Research 104, 419-430.

Livingstone, M.S. \& Hubel, D.H. (1982). Thalamic inputs to cytochrome oxidase-rich regions in monkey visual cortex. Proceedings of the National Academy of Sciences of the U.S.A. 79, 6098-6101.

Livingstone, M.S. \& Hubel, D.H. (1983). Specificity of cortico-cortical connections in monkey visual system. Nature 304, 531-534.

Livingstone, M.S. \& Hubel, D.H. (1984a). Anatomy and physiology of a color system in the primate visual cortex. Journal of Neuroscience $\mathbf{4}$, 309-356.

Livingstone, M.S. \& Hubel, D.H. (1984b). Specificity of intrinsic connections in primate primary visual cortex. Journal of Neuroscience 4, 2830-2835.

Livingstone, M.S. \& Hubel, D.H. (1987). Connections between layer 4B of area 17 and the thick cytochrome oxidase stripes of area 18 in the squirrel monkey. Journal of Neuroscience 7, 3371-3377.

Livingstone, M.S. \& Hubel, D.H. (1988). Segregation of form, color, movement, and depth: anatomy, physiology, and perception. Science 240, 740-749.

Lund, J.S., Hendrickson, A.E., Ogren, M.P. \& Tobin, E.A. (1981). Anatomical organization of primate visual cortex area VII. Journal of Comparative Neurology 202, 19-45.

Malach, R., Tootell, R.B.H. \& MaloneK, D. (1994). Relationship between orientation domains, cytochrome oxidase stripes, and intrinsic horizontal connections in Squirrel monkey area V2. Cerebral Cortex 4, $151-165$.

Movshon, J.A. \& Newsome, W.T. (1996). Visual response properties of striate cortical neurons projecting to area MT in macaque monkeys. Journal of Neuroscience 16, 7733-7741.

Munk, M.H., Nowak, L.G., Girard, P., Chounlamountri, N. \& BulLIER, J. (1995). Visual latencies in cytochrome oxidase bands of macaque area V2. Proceedings of the National Academy of Sciences of the U.S.A. 92, 988-992.

Nakamura, M., Gattass, R., Desimone, R. \& Ungerleider, L.G. (1993). The modular organization of projections from areas V1 and V2 to areas V4 and TEO in macaques. Journal of Neuroscience 13, 3681-3691.

Nealey, T.A. \& Maunsell, J.H.R. (1994). Magnocellular and parvocellular contributions to the responses of neurons in macaque striate cortex. Journal of Neuroscience 14, 2069-2079.

Neltner, L., Hansel, D., Mato, G. \& Meunier, C. (2000). Synchrony in heterogenous networks of spiking neurons. Neural Computation 12, $1607-1641$

Ogren, M.P. \& Hendrickson, A.E. (1977). The distribution of pulvinar terminals in visual areas 17 and 18 of the monkey. Brain Research 137, $343-350$. 
Olavarria, J.F. \& VAN EsSEn, D.C. (1997). The global pattern of cytochrome oxidase stripes in visual area V2 of the macaque monkey. Cerebral Cortex 7, 395-404.

Orban, G.A., Kennedy, H. \& Bullier, J. (1986). Velocity and direction selectivity of neurons in areas V1 and V2 of the monkey: Influence of eccentricity. Journal of Neurophysiology 56, 462-480.

Peterhans, E. \& von der Heydt, R. (1993). Functional organization of area V2 in the alert macaque. European Journal of Neuroscience 5, 509-524.

Peters, A., Cifuentes, J.M. \& Sethares, C. (1997). The organization of pyramidal cells in area 18 of the rhesus monkey. Cerebral Cortex 7, 405-421.

Purves, D., Shimpi, A. \& Lotto, R.B. (1999). An empirical explanation of the Cornsweet effect. Journal of Neuroscience 19, 8542-8551.

Raiguel, S., Van Hulle, M.M., XiaO, D.K., Marcar, V.L. \& Orban, G.A. (1995). Shape and spatial distribution of receptive fields and antagonistic motion surrounds in the middle temporal area (V5) of the macaque. European Journal of Neuroscience 7, 2064-2082.

RocKLAND, K.S. (1985). A reticular pattern of intrinsic connections in primate area V2 (area 18). Journal of Comparative Neurology 235, 467-478.

RocKLAND, K.S. (1994). The organization of feedback connections from area V2 (18) to V1 (17). In Primary Visual Cortex in Primates, ed. Peters, A. \& Rockland, K.S., pp. 261-299. New York: Plenum.

Rockland, K.S. \& PANDYA, D.N. (1979). Laminar origins and terminations of cortical connections of the occipital lobe in the rhesus monkey. Brain Research 179, 3-20.

Rockland, K.S., SAleEM, K.S. \& TANaKa, K. (1994). Divergent feedback connections from areas V4 and TEO in the macaque. Visual Neuroscience 11, 579-600.

RocKLAND, K.S. \& VIRGA, A. (1990). Organization of individual cortical axons projecting from area V1 (area 17) to V2 (area 18) in the macaque monkey. Visual Neuroscience 4, 1-28.

RoE, A.W. \& Ts'o, D.Y. (1995). Visual topography in primate V2: multiple representation across functional stripes. Journal of Neuroscience 15, 3689-3715.

Roe, A.W. \& Ts'o, D.Y. (1997). The functional architecture of area V2 in the macaque monkey. In Cerebral Cortex Vol. 12: Extrastriate Visual Cortex in Primates, ed. Rockland, K.S., KaAs, J.H. \& Peters, A., pp. 295-333. New York: Plenum Press.

Sary, G., Vogels, R. \& Orban, G.A. (1993). Cue-invariant shape selectivity of macaque inferior temporal neurons. Science 260, 995-997.

Schillen, T.B. \& Konig, P. (1994). Binding by temporal structure in multiple feature domains of an oscillatory neuronal network. Biological Cybernetics 70, 397-405.

Shadlen, M.N. \& Movshon, J.A. (1999). Synchrony unbound: A critical evaluation of the temporal binding hypothesis. Neuron 24, 67-77, $111-25$.

SHIPP, S. (1995). The odd couple. Current Biology 5, 124-128.

SHIPP, S. \& ZEKI, S. (1985). Segregation of pathways leading from area V2 to areas V4 and V5 of macaque monkey visual cortex. Nature $\mathbf{3 1 5}$, $322-325$

SHIPP, S. \& ZEKI, S. (1989a). The organization of connections between areas V5 and V1 in macaque monkey visual cortex. European Journal of Neuroscience 1, 309-332.

SHIPP, S. \& ZEKI, S. (1989b). The organization of connections between areas V5 and V2 in macaque monkey visual cortex. European Journal of Neuroscience 1, 333-354.

SHIPP, S. \& ZEKI, S. (2002). The functional organization of area V2. II: The impact of stripes on visual topography. Visual Neuroscience (submitted).

Singer, W. (1998). Consciousness and the structure of neuronal representations. Philosophical Transactions of the Royal Society B (London) 353, 1829-1840.

Singer, W. (1999). Neuronal synchrony: A versatile code for the definition of relations? Neuron 24, 49-65, 111-25.

Singer, W. \& Gray, C.M. (1995). Visual feature integration and the temporal correlation hypothesis. Annual Review of Neuroscience $\mathbf{1 8}$, $555-586$.

SoKal, R.R. \& Rohlf, F.J. (1995). Biometry (3rd edition) New York: Freeman.

Stromeyer, C.F., III, Kronauer, R.E., Ryu, A., Chaparro, A. \& Eskew, R.T., JR. (1995). Contributions of human long-wave and middle-wave cones to motion detection. Journal of Physiology 485, 221-243.

Tamura, H., Sato, H., Katsuyama, N., Hata, Y. \& Tsumoto, T. (1996).
Less segregated processing of visual information in V2 than V1 of the monkey visual cortex. European Journal of Neuroscience 8, 300309.

Thorell, L.G., DeValois, R.L. \& Albrecht, D.G. (1984). Spatial mapping of monkey V1 cells with pure color and luminance stimuli. Vision Research 24, 751-769.

Tononi, G., Sporns, O. \& Edelman, G.M. (1992). Re-entry and the problem of integrating multiple cortical areas: Simulation of dynamic integration in the visual system. Cerebral Cortex 2, 310-335.

Tootell, R.B.H., Silverman, M.S., DeValois, R.L. \& Jacobs, G.H. (1983). Functional organization of the second cortical area in primates. Science 220, 737-739.

Tootell, R.B.H., Hamilton, S.L. \& Switkes, E. (1988a). Functional anatomy of macaque striate cortex. IV. Contrast and magno parvo streams. Journal of Neuroscience 8, 1594-1609.

Tootell, R.B.H., Silverman, M.S., Hamilton, S.L., DeValois, R.L. \& SwITKES, E. (1988b). Functional anatomy of macaque striate cortex. III. Color. Journal of Neuroscience 8, 1569-1593.

Tootell, R.B.H., Silverman, M.S., Hamilton, S.L., Switkes, E. \& DeValois, R.L. (1988c). Functional anatomy of macaque striate cortex. V. Spatial frequency. Journal of Neuroscience 8, 1610-1624.

Tootell, R.B.H. \& Hamilton, S.L. (1989). Functional anatomy of the second visual area (V2) in the macaque. Journal of Neuroscience $\mathbf{9}$, 2620-2644.

Treisman, A. (1996). The binding problem. Current Opinion in Neurobiology 6, 171-178.

Ts'o, D.Y., Frostig, R.D., Lieke, E.E. \& Grinvald, A. (1990). Functional organization of primate visual cortex revealed by high resolution optical imaging. Science 249, 417-420.

Ts'o, D.Y. \& GilberT, C.D. (1988). The organization of chromatic and spatial interactions in the primate striate cortex. Journal of Neuroscience 8, 1712-1727.

Van Essen, D.C., Newsome, W.T., Maunsell, J.H.R. \& Bixby, J.L. (1986). The projections from striate cortex to areas V2 and V3 in the macaque monkey: Asymmetries, areal boundaries and patchy connections. Journal of Comparative Neurology 244, 451-480.

Ware, C. \& Cowan, W.B. (1983). The chromatic Cornsweet effect. Vision Research 10, 1075-1077.

WELlER, R.E. \& KAAS, J.H. (1983). Retinotopic pattern of connections of area 17 with visual areas V-II and MT in macaque monkeys. Journal of Comparative Neurology 220, 253-279.

Wong-Riley, M.T. \& Carroll, E.W. (1984). Quantitative light and electron microscopic analysis of cytochrome oxidase-rich zones in V-II prestriate cortex of the squirrel monkey. Journal of Comparative Neurology 222, 18-37.

Wong-RiLeY, M.T.T. (1979). Changes in the visual system of monocularly sutured or enucleated cats demonstrable with cytochrome oxidase histochemistry. Brain Research 171, 11-28.

XiaO, Y., Zych, A. \& Felleman, D.J. (1999). Segregation and convergence of functionally defined V2 thin stripe and interstripe compartment projections to area V4 of macaques. Cerebral Cortex 9 , 792-804.

YoshioKA, T., LeVITT, J.B. \& Lund, J.S. (1994). Independence and merger of thalamocortical channels within macaque primary visual cortex: Anatomy of interlaminar projections. Visual Neuroscience 11, 467-489.

Yoshioka, T., Blasdel, G.G., Levitt, J.B. \& Lund, J.S. (1996). Relation between patterns of intrinsic lateral connectivity, ocular dominance, and cytochrome oxidase-reactive regions in macaque monkey striate cortex. Cerebral Cortex 6, 297-310.

YoshiokA, T. \& Dow, B.M. (1996). Color, orientation and cytochrome oxidase reactivity in areas V1, V2 and V4 of macaque monkey visual cortex. Behavioral Brain Research 76, 71-88.

ZEKI, S. (1983). Colour coding in the cerebral cortex: the reaction of cells in monkey visual cortex to wavelengths and colors. Neuroscience $\mathbf{9}$, 741-765.

ZEKI, S. \& SHIPP, S. (1988). The functional logic of cortical connections. Nature 335, 311-317.

ZeKI, S. \& SHIPP, S. (1989a). Functional segregation within area V2 of macaque monkey visual cortex. In Seeing Contour and Colour, ed. Kulikowski, J.J., Dickinson, C.M. \& Murray, I.J., pp. 120-124. Oxford: Pergamon.

ZEKI, S. \& SHIPP, S. (1989b). Modular connections between areas V2 and V4 of macaque monkey visual cortex. European Journal of Neuroscience 1, 494-506. 